\title{
Measurements of the horizontal sprayer boom movements by sensor data fusion
}

\author{
D. Ooms ${ }^{\text {a }}$, F. Lebeau ${ }^{\text {a }}$, R. Ruter ${ }^{\text {a }}$, M.-F. Destain ${ }^{\text {a }}$ \\ ${ }^{a}$ Gembloux Agricultural University, Passage des Déportés 2, \\ B-5030 Gembloux, Belgium
}

\begin{abstract}
A study investigated the horizontal movements of trailed sprayer booms with the aim of distinguishing their yaw and jolt motions as well as their deformations. Two vehicles were equipped with a radar speed sensor and a three-axis dynamic measurement unit, while each boom was instrumented with ultrasonic sensors and accelerometers. Sensor fusion was used to extract yaw, jolt and deformation speeds by assuming that the motion of any point of the boom is equal to the superimposition of complementary high and low-frequency motions, delimited by a cut-off frequency, estimated on the basis of laboratory trials. Tests performed on trailed sprayers equipped with 22 and $24 \mathrm{~m}$ boom lengths, on different soils, indicated that yaw, jolting and deformation respectively occurred near $0.3 \mathrm{~Hz}, 2 \mathrm{~Hz}$ and $1 \mathrm{~Hz}$. Velocity variations relative to the forward speed of the vehicle were lower than $0.57 \mathrm{~m} / \mathrm{s}$ and were reached at a speed of $3.5 \mathrm{~m} / \mathrm{s}(12.7 \mathrm{~km} / \mathrm{h})$. Ground deposit, roughly estimated as proportional to the inverse of the speed, exhibited coefficients of variation between 4 and $6 \%$ for all tests.
\end{abstract}

Keywords: Spray boom movements ; Sensors; Sensor fusion ; Field measurements

\section{Introduction}


The use of chemical plant protection products is important today and will continue to be so in the foreseeable future to secure food for the population at reasonable prices. Apart from the use of other plant protection methods (such as integrated plant protection), chemical plant protection methods are the most frequently used today since they are efficient, easy to employ and show a broad spectrum of application with regard to controlling pests, pathogens, weeds, stalk length and liquid fertilizers application. They remain necessary in the presence of severe infestation with harmful organisms that require massive action because, for example, special weather conditions occur, resistance is broken down, mutants or new pathogens occur. Nevertheless, the greater awareness of public opinion regarding environment protection and the increasing cost of chemicals require more precise spraying methods. The accuracy of distribution depends on nozzle characteristics, pressures variation in hydraulic equipment, wind and drift and is also influenced to a large extent by boom movements. Vertical movements of the boom affect the deposit density both along and across the vehicle's tracks, due to the changing spread of the spray with changing height. Variations in the horizontal component of the velocity of the boom cause fluctuations in the deposit density along the track. The direct source of the boom movements are the frame movements. These are induced by the unevenesses of the ground and quick variations of the forward speed of the hauling vehicle. The most important device used to reduce the boom movements is the suspension located between the frame and the boom, which is designed to isolate the boom from the roll motion of the sprayer frame. Trapezoïdal and pendular forms (or a combination) are the most common designs. Some machines are provided with specially designed suspension systems located between the wheel axle(s) and the frame. Sometimes, an horizontal suspension is added to isolate the boom from the yaw and jolting movements of the frame. 
The techniques developped to evaluate boom movements and deformations in field conditions can be divided into absolute and relative measurement methods. The most widely used absolute method consisted in using small seismic accelerometers fastened to the sprayer. This method has the advantage that boom behaviour is not modified by the measuring technique. Furthermore, it can be readily used on any sprayers at work in the field, in order to compare them. The technique developed by Nation (1982) to measure the height variations of the boom sprayer consisted in computing displacements by double integration of accelerometer data. The main problem encountered in this method is the difficulty to find the cut-off frequency of the appropriate filter to remove any long-term trends or drifts inherent to accelerometer outputs. Another absolute method developed to overcome the limitations of the accelerometer method is a semi-automated method using a video camera (Lardoux et al., 1998). In this method, however, data were only provided over small distances (5 to $10 \mathrm{~m}$ ) due to the limited view angle of the camera and on-line implementation was not possible. Thirdly, a method based on a fixed laser sensor was developed by Vannuci et al. (1992) but it, too, provided measurements only over a limited distance. Relative displacement methods use the vehicle as the datum. In the method proposed by Lebeau and Destain (1998), the sensor was a laser distance meter mounted on the boom, having its beam directed to a special reflective target fixed rigidly at the front of the tractor and oriented perpendicular to the beam. The main drawback of this method was its tedious character due to the need to position the target correctly before the trials.

The aim of this paper is to present a suitable technique for indicating directly the absolute horizontal speeds of spray booms which have been shown to have a significant effect on the density of the spray deposit (Speelman and Jansen, 1974, Sinfort et al., 1997, Jong et al, 2000). Particularly, the measurements will be useful to compare the efficiency of the numerous types of suspensions developed by the manufacturers. Within this scope, the boom 
movements, including rigid body motion (yaw and jolting) and boom deformations, should be distinguished. This implies the acquisition of information in a frequency range from about 0.1 $\mathrm{Hz}$ to $12.5 \mathrm{~Hz}$, which covers the frequency spectrum of boom vibrations (De Baerdemaker et al., 1983). Furthermore, absolute movement of the boom is desired in order to facilitate comparisons of different boom designs. At last, the measurement method should be applicable to any sprayer model: mounted (Fig. 1a), trailed (Fig. 1b) or self-propelled (Fig. 1c).

\section{Method}

The method aims to measure the yaw, jolting and deformation movements of the boom at several points (Fig. 2). The steps involved in the evaluation of the absolute horizontal boom movements are shown in Fig. 3. The sensors were accelerometers, distance-meters, a radar speed meter and a 'dynamic measurement unit'. Indeed, in the absence of a unique sensor covering the $0-12.5 \mathrm{~Hz}$ frequency range, it was necessary to use accelerometers (suited to high frequency measurement) and distancemeters (appropriate at low frequency measurement) in combination in order to achieve acceptable results The radar speed meter and the dynamic measurement unit (that provided the rotation speed about a vertical axis) were used to measure the absolute vehicle motion. A detailed description of the sensors is given in section 3. The implementation of the sensors on a trailed spraying machine is shown on Fig. 4. The dynamic measurement unit excepted, all sensors produced continuous data as a function of time. These data were processed to extract several vectors $x_{i}(t)$, which represented each a part $\mathrm{i}$ of the position $\mathrm{x}$ of a single point located on the boom as a function of time $t$. The extraction of each vector $\mathrm{x}_{\mathrm{i}}(\mathrm{t})$ is described in section 4. In our reference frame, the $\mathrm{X}$ axis is the forward direction of the tractor. We suppose that the sprayer trajectory and the boom orientation are respectively parallel and perpendicular to the direction of the field tracks. The 
direction of each individual measurement may be slightly different, but as the distortion does not exceed a few degrees ( 1 to 5 degrees for the yaw movement), the influence on the final results is negligible. The origin of the $\mathrm{X}$ axis may be placed anywhere as only the speed data is interpreted. It may be, for example, the starting position of the boom centre.

Processing included transformations from the time to the frequency domain and vice versa. The next step was to perform a fusion of the feature vectors to generate a database representative of the boom movements: yaw, jolting and boom deformation. The fusion method, that consists in a single addition of the feature vectors $\mathrm{x}_{\mathrm{i}}(\mathrm{t})$, was based on the following premises:

1) The motion of any point on the boom is equal to the superimposition of complementary high and low frequency motions, delimited by a cut-off frequency. This operation is performed in the frequency domain.

2) The absolute motion of any point on the boom is equal to the sum of its motion relative to the vehicle and of the vehicle movement. This operation is performed in the time domain.

The other assumptions were:

3) The frame of the sprayer is a rigid body.

4) The motion of the centre of the boom is the jolting motion. It is affected neither by yaw or by deformation.

5) Deformation occurs at higher frequencies than yaw motion.

6) The vertical and the horizontal motions are uncoupled. 


\section{Sensors selection and dynamic calibration}

The sensors could be classified as follows:

- Sensors for measuring absolute vehicle motion

The radar speed sensor (RGSS-201, Philips Automotive Electronics Co.), measuring the vehicle forward speed, was located on the sprayer frame. It generates impulses corresponding to a covered distance of 1/128 m. A six axis dynamic measurement unit (DMU) (Crossbow Technology) was located on the frame of the boom and measured its yaw angular rate.

- $\quad$ Sensors measuring the relative motion between the boom and the frame Two ultrasonic sensors US1 and US2 (BANNER T30UUPAQ) were mounted on the right and left parts of the boom, at $1.2 \mathrm{~m}$ from the centre. Their measuring range was from 150 to $1000 \mathrm{~mm}$. Suitable targets were located on the sprayer frame. Some others distance-meters (such as infrared or laser sensors) could be used, but ultrasonic sensors were preferred due to their low cost and their convenient measuring range.

- Sensors measuring the absolute motion of the boom

Five capacitive accelerometers AC1 to AC5 were used, one of them being fixed on each part of the boom. In the central and middle positions, their sensitivity was $500 \mathrm{mV} / \mathrm{g}$ (CXLO4M3, Crossbow Technology) and reached $1000 \mathrm{mV} / \mathrm{g}$ on the external parts (CXL02LF3, Crossbow Technology).

The sensors were calibrated in order to verify the relationship between the physical value and the delivered voltage. Furthermore, information on the dynamic aspects of their use was required in our particular application. Their frequency response needed to be ascertained and especially it was necessary to find the cut-off frequency to introduce in the fusion sensors 
process. This cut-off frequency establishes the limit between the correct usage of the sensor and the domain where the signal has a poor gain or is corrupted. As the output of accelerometers is affected by an offset error which is slowly varying with time (because of thermal drift), only the high-frequency output of these sensors was used. On the other hand, distance-meters are more appropriate to measurements comprised from dc to several Hz. To determine the cut-off frequencies, the following set-up was designed (Fig. 5.): two accelerometers (one of each model) and one ultrasonic sensor were mounted together on a rigid beam. The beam was mounted on a displacement table by means of linear guides (linear module MLFI25056ZR4000-3500, INA Roulements S.A.) and moved by an electrical motor (HDY 115-E6-130S), which was computerized. This device could impose any motion defined by the user, with a maximum speed of $2 \mathrm{~m} / \mathrm{s}$ and a maximum acceleration of $10 \mathrm{~m} / \mathrm{s}^{2}$. Sinusoidal motions were successively imposed to the beam, at frequencies ranging from 0.05 to $5 \mathrm{~Hz}$. The amplitude of each sine wave was measured independently twice to verify that the command was correctly executed. The first measurement was made using a graduated ruler. The second measurement was carried out using a laser sensor (DME2000, Sick Optic Electronics), which is reliable from DC to $13 \mathrm{~Hz}$. The two measurements offered a precision of $+-1 \mathrm{~mm}$ and agreed in all cases. The experiment results are shown in Fig. 6. The two accelerometers had unsatisfactory gain below $0.1 \mathrm{~Hz}$, while the ultrasonic sensor had a good reliability at all the tested frequencies. Although ultrasonic distance sensors can provide valid data at high frequencies, the use of accelerometers remained necessary for three reasons:

1) The measurement of the deformation motion requires several sensors distributed along the boom, which is not possible with distance sensors.

2) During the field tests, spectral analysis of the distance meters revealed that they were affected by boom deformation. As the distance sensors were used to measure the yaw motion, the high frequency part of the distance signals (corresponding to the boom deformation 
frequencies) had to be removed to avoid misinformation and only the lower frequencies were used. Thus accelerometers remain necessary to measure the boom yaw motion, when the frequency of this motion is above $0.2 \mathrm{~Hz}$ (a linear interpolation between the five positions given by the accelerometric data was used to distinguish the yaw motion from the deformation motion above $0.2 \mathrm{~Hz}$, see below).

3) The behaviour of the radar sensor is unknown at high frequencies and estimation of the frame jolting by this means is hazardous. It may be affected by the soil roughness and vertical vibrations of the vehicle. A test was made to verify the reliability of the DC part of the signal (mean forward speed), but this sensor was not used to measure vibrations above $0.2 \mathrm{~Hz}$. As the data of the radar sensor was fused with that of distance sensors, the two sensors must cover the same frequency range $(0-0.2 \mathrm{~Hz})$. An accelerometric sensor was required to measure the jolting motion above $0.2 \mathrm{~Hz}$.

As thermal drift could be the main source of accelerometric measurement error, a second experiment was performed to measure the error in severe temperature conditions. The accelerometers were fixed to a frame placed outside in a sunny and windy place. The signal of each of them was recorded for $20 \mathrm{~s}$ (as in the subsequent field tests). The linear value of each signal was extracted to remove the error due to the possible frame slope and the nonvibratory part of the signal. Then the remaining signal was integrated once to obtain the speed signal. As the sensors were intended to measure the horizontal speed of sprayer booms, the error on the measured speed had to stay within an acceptable range. On the tested sprayers, the amplitudes of the horizontal boom velocities were between 0 and $0.4 \mathrm{~m} / \mathrm{s}$. These values were obtained from estimations made before fixing the parameters of the method such as the cut-off frequency, the filtering methods, and the type of sensors. Allowing an error level of 5 $\%$ on the mean amplitude, the maximum error must stay below $10 \mathrm{~mm} / \mathrm{s}$ for each trial. The 
speed signal was filtered using a high pass filter at five different cut-off frequencies $(0.05,0.1$, $0.15,0.2$ and $0.25 \mathrm{~Hz}$ ). The maximum value of the remaining error was compared to the allowance. Fifteen repetitions were made at three different sites. Results showed that the minimum cut-off frequency of the high-pass filter was $0.15 \mathrm{~Hz}$ for a maximum error of $5 \%$. Increasing the cut-off frequency to $0.2 \mathrm{~Hz}$ reduced the error to $4 \%$ (Fig. 7). The cut-off frequency was therefore fixed to $0.2 \mathrm{~Hz}$ to eliminate most of static and dynamic errors.

To verify the dynamic validity range of the DMU, a tilt sensor was used. The two sensors were mounted together on the sprayer frame and the roll motion was recorded while the sprayer was running in a field. The DMU sensor provided valid data from $0 \mathrm{~Hz}$ to $0.2 \mathrm{~Hz}$, and a only very small amount of drift was observed after integration of the angular rate signal. The DMU may be used to measure angular displacement below the cut-off frequency of $0.2 \mathrm{~Hz}$.

The data provided by the radar sensor on a wheat field (height: $75 \mathrm{~cm}$, very uneven surface) was processed to extract the distance covered by the sprayer. The same distance was measured using a theodolithe. The error of the radar measurement was approximatively $1 \%$.

\section{Data acquisition, preprocessing and processing}

The DMU and laser sensor (used for sensor calibration and method validation) delivered discrete data in function of time to the RS-232 serial port of the computer. Other sensors delivered analogue signals. They were acquired with an acquisition board (DAQCard-AI-16E- 
4, National Instruments) located in a laptop (Fig. 8) at a sample rate of $1000 \mathrm{~Hz}$. The acquisition was performed by developing a Labview (National Instruments) application. In the preprocessing step (Fig. 9a-9c), the signals were formatted to fit together (sample rate equal to $1 \mathrm{kHz}$, same period of $20 \mathrm{~s}$ and units). The data format used to process the sensor fusion was the absolute displacement of a unique point located on the boom, in metres. The preprocessing step included:

- the transformation of the voltage data to accelerations (accelerometers), distances (ultrasonic sensors), rotation speed (DMU) and covered distance (radar);

- the reduction of the length of each signal to 20 seconds, corresponding to the middle of the trial;

- the increasing of the sample rate of the DMU sensor up to $1000 \mathrm{~Hz}$ using a zero-degree interpolation.

In the processing step, signals were first transformed in the frequency domain by a Fourier Transform with Matlab (Mathworks) in order to filter them. The use of the Fourier Transform ensured that the bandpasses of the two signals did not contain the same frequencies. The linear part of each signal was removed, but recorded to be restored afterwards if necessary. Then the Fast Fourier Transform was applied. The two indexes corresponding to the cut-off frequency were marked, and the values corresponding to unwanted frequencies were removed, respectively inside or outside the marked indexes for a low-pass or high-pass filter. The Inverse Fourier Transform was applied to obtain the filtered signal. The cut-off frequency used was $0.2 \mathrm{~Hz}$, according to the calibration explained in section 3 . The last step of the processing depended on the type of sensor and its location on the vehicle, and is detailed in next paragraph. 


\subsection{Yaw data processing}

The information at low frequencies (lower and equal to $0.2 \mathrm{~Hz}$ ) was provided by the DMU (Fig. 9c) and the ultrasonic sensors US1 and US2 (Fig. 9b). The DMU sensor was used to measure the yaw of the frame. The linear part of the signal was removed and a low-pass filter was applied, according to the procedure explained above. The signal was integrated once to obtain the angular displacement of the frame. By multiplying the angle tangent by the distance of the selected point from the boom centre, the displacement of the point due to the frame's yaw was obtained:

$$
Y a w C=d 1 \times \tan (R O T)
$$

d1: distance between the centre of the boom and the point of interest ROT: yaw angle of the frame

At low frequencies, the signals US1 and US2 were processed according to:

$$
Y a w B=d 1 \times \frac{U S 1-U S 2}{d 2}
$$

d2: distance between the two ultrasonic sensors

US1: distance measured by the left ultrasonic sensor

US2: distance measured by the right ultrasonic sensor

At higher frequencies, the signals of the five accelerometers AC1 to AC5 were filtered with a high-pass filter (see above) and integrated twice to obtain the displacement signals of each of 
the five instrumented points. As these signals include rigid body motion and boom deformation, the latter was removed by applying a linear regression on the five instrumented points (Fig. 9a). The central point was set to zero and the central accelerometric signal was substracted from the other signals to calculate the parameters of the regression, according to the second hypothesis ( section 2.1). As the number of accelerometers was limited to five, the result is not an exact measurement of the yaw motion, but an estimation. A small amount of deformation may be measured as yaw, as well as some yaw may be measured as a deformation. It was necessary to take this into account in the interpretation.

\subsection{Jolting data processing}

In order to evaluate the jolting motion in the low frequency domain $(\leq 0.2 \mathrm{~Hz})$, the radar sensor and the ultrasonic sensors were used. The radar sensor measured the absolute jolting of the frame, as well as the forward speed (Fig. 9c). The mean value between the two ultrasonic signals US1 and US2 was used to estimate the relative jolting between the boom and the frame (Fig. 9b):

Jolting $B=-\frac{U S 1+U S 2}{2}$

The high-frequency portion $(>0.2 \mathrm{~Hz})$ of the central accelerometer AC3 signal was integrated twice to obtain the absolute jolting vibration of the boom (AC3,DE3, Jolting A, Fig. 9a). 


\subsection{Deformation data processing}

The boom deformation was estimated using the accelerometers, assuming that it was limited to frequency higher than $0.2 \mathrm{~Hz}$. The deformation motion was obtained from the data of a single accelerometer, located at the point whose motion is calculated (gray-shaded, Fig. 9a). After subtracting the motions due to jolting and yaw, the remaining part of the signal was considered to be the boom deformation. The deformation may be estimated at other places, but not exactly. It could be done, for example, by means of a linear interpolation.

\subsection{Sensor fusion}

The sensor fusion required only a single addition. The three components of the yaw motion were summed to obtain the full boom yaw motion in the time domain. The three components of the jolting motion were summed to obtain the full boom jolting motion. The whole fusion process in shown in Fig. 10. The yaw, jolting and deformation motions were additionned to the mean vehicle speed to obtain the full motion of the boom at a single point. The result is the point displacement. The speed of the specified point is obtained by differentiating once the displacement signal. The entire process can be repeated to obtain the motion of another point. It is important to notice that the calculation of the total motion above $0.2 \mathrm{~Hz}$ depended only on the single accelerometric signals, and may be obtained independently from the fusion process.

\section{Field tests}




\subsection{Validation of the method}

The proposed method has been compared to a laser method similar to that used by Vannucci (1992). In our case, the laser beam was not scanning the target, this latter was fixed vertically on the ground while the laser sensor was fixed horizontally on the boom (Fig. 11). This arrangement allows only the measurement of the horizontal boom movement at a single point at a distance of 10 metres. The laser sensor (DME 2000, Sick Optic Electronics) was fixed to the left of the boom of a mounted sprayer, at the same place as the most external accelerometer. Two experiments were made: on a fallow and on a very uneven meadow. The forward speed of the tractor was approximatively $1.5 \mathrm{~m} / \mathrm{s}$. Results on fallow are shown in Fig. 12. The two measurement methods are compared (all frequencies except $0 \mathrm{~Hz}$ ). The broken line corresponds to the laser signal acquired at a sample rate equal to $12.5 \mathrm{~Hz}$.

The results obtained by both methods are rather similar, both in frequency and in amplitude.

\subsection{Tests on trailed machines}

Trials were performed in July and October 2000 (Table 1). Fields with different topographies and textures were chosen to meet varied working conditions. Two trailed sprayers were tested in two different soil conditions and crops. The main parameters were the amount of liquid in the tank and the forward speed. Two available machines, designed by different manufacturers, were tested. They both have trapezoïdal suspensions. The differences were the materials used for the contact between the boom and the sprayer frame (blocks for sprayer 1, rolls for sprayer 2) and the dimensions of the trapeze. The trapeze of sprayer 1 was $570 \mathrm{~mm}$ long at the bottom, $385 \mathrm{~mm}$ long at the top and $280 \mathrm{~mm}$ high. The trapeze of sprayer 2 was 
$1824 \mathrm{~mm}$ long at the bottom, $1380 \mathrm{~mm}$ long at the top and $355 \mathrm{~mm}$ high. Neither of these sprayers have especially designed horizontal boom or frame suspensions.

\subsubsection{Characterization of the vibration modes}

The horizontal speed of a single point located at $8.5 \mathrm{~m}$ to the right of the centre of the boom was calculated in the time and frequency domains. The spectrum of the horizontal speed gave information about the vibration modes. Figs. 13, 14 and 15 show the yaw, jolting and deformation spectra of sprayer 1 for each forward speed. Figs. 16 and 17 show the yaw and deformation spectra of sprayer 2 for each forward speed. The power spectral density was not used, since high-frequency vibrations of high energy do not involve significant speed amplitudes for either machine. No significant vibration mode was observed above $5 \mathrm{~Hz}$.

- Concerning yaw, the behaviour of the two machines is rather similar: the yawing frequency is located around $0.3 \mathrm{~Hz}$ (Table 2). In both cases, the influence of the forward speed is obvious: the amplitude of the yawing motion increases with speed. Table 3 presents the variance of the instantaneous speed.

- Concerning jolting, for sprayer 1, the main frequencies were respectively 2 and $1.65 \mathrm{~Hz}$ for 9 and $11 \mathrm{~km} / \mathrm{h}$. The very-low frequency jolting motion had a very high amplitude, due to the non-constant forward speed of the tractor which depended on the vehicle's driver. When the amplitudes were too low (jolting modes of sprayer 2), no interpretation of the vibration modes could be made.

- The main deformation occurs at frequencies almost similar (between $1 \mathrm{~Hz}$ and $2 \mathrm{~Hz}$ ) for both machines, although machine 2 exhibits several deformation modes at higher speeds.

\subsubsection{Amplitudes of the vibrations and influence of the motion on the ground deposit}


Table 3 indicates for each trial

- the variance of the velocities at the specified point (the mean value being the forward speed);

- the coefficient of variation;

- the maximum amplitude of velocity.

The jolting motion below $0.2 \mathrm{~Hz}$ was not included in the calculation of the total amplitude, since its interpretation is difficult :

1) The jolting motion includes both sprayer and tractor response.

2) The low-frequency jolting motion of the tractor is compensated by the modification of the flow rate at the pump (the pump is motored by the tractor engine). This motion does not significantly influence the spray deposit.

3) The amplitude low frequency jolting motion $(0-0.2 \mathrm{~Hz})$ is much higher than at high frequencies $(>0.2 \mathrm{~Hz})$. It could exceed twice the amplitude of the yaw motion at the boom tip as the driver does not maintain a constant speed.

In conclusion, including the low-frequency jolting motion in the calculation of the boom motions in relationship with the spray deposit would be a mistake.

The maximum amplitude of the speed variation was $0.24 \mathrm{~m} / \mathrm{s}$, for a mean forward speed of $1.67 \mathrm{~m} / \mathrm{s}(6 \mathrm{~km} / \mathrm{h})$, and $0.64 \mathrm{~m} / \mathrm{s}$, for a mean forward speed of $3.5 \mathrm{~m} / \mathrm{s}(12.7 \mathrm{~km} / \mathrm{h})$. That represents relative variations of $14 \%$ and $18 \%$ on the total horizontal speed. These values are 
much lower than those evaluated by Nation (1982), but the machines at that time were very different (boom rigidly attached to the sprayer frame). Nevertheless, these results show that actual sprayers are substantially better on that score. For other parameters, such as the fill rating of the tank, the field and soil conditions, and the sprayer, no conclusion may be made. The differences observed on the speed variance were not significant, due to high variations between the repetitions of the trials. The variation of the ground deposit increases with the boom velocities but decreases rank with the absolute forward speed. Assuming that the ground deposit is proportional to the inverse of the speed, the coefficient of variation of the ground product distribution, due to the horizontal motions, was calculated according to

$$
C V=\frac{\frac{1}{n} \cdot \sum_{i=1}^{n}\left[1 / V_{i}-\operatorname{mean}\left(1 / V_{i}\right)\right]}{\operatorname{mean}\left(1 / V_{i}\right)}
$$

n: distance (in inches) covered during the trial $V_{i}:$ forward speed measured at $i$ inches from the starting position

The coefficient of variation is sensitive to the distance unit used: increasing the step size decreases the numbers of steps for the same covered distance, decreasing the coefficient of variation. When the step size increases, the variances between the steps decreases, because the residual variances (inside steps) are not taken into account. (Dagnelie, 1975). There is no common value fixed for that purpose, but in static spray pattern evaluation, the value of $5 \mathrm{~cm}$ is commonly used. Very low values are not useful because of the spray thickness (space including $90 \%$ of the static spray pattern in the forward direction) that eliminates the smallest variations in the spray deposit. Common spray thicknesses are around $10 \mathrm{~cm}$ ( for Teejet 11003 VK flat spray nozzle located at $70 \mathrm{~cm}$ above the ground, $\mathrm{p}=3 \mathrm{bar}$ ) or $12 \mathrm{~cm}$ (at $50 \mathrm{~cm}$ 
above the ground, $p=2$ bar). The value of the distance step should be less than the spray thickness, but not too much. The chosen value is not optimised. In our case, the predicted coefficient of variation of the ground deposit is nearly the same as the coefficient of variation of the horizontal speed (the absolute difference is lower than $1 \%$ ).

Results are shown in Table 4 for both sprayers. The increase of the total speed compensated the increase of the speed variance in both cases. For sprayer 1, when the forward speed was multiplied by 1.22 , the boom speed variance was multiplied by 2.06 and the standard deviation by 1.44 (Table 3), but the coefficients of variation of the total speed and of the ground deposit were multiplied respectively by 1.18 and 1.11 . (Tables 3,4 ).

\section{Conclusion}

The proposed method is well-suited to measure the horizontal boom movements of sprayers in the field. Our experience has shown that environmentally suitable equipment (reliable sensors, robust computer and cables) is essential. The results are reliable when compared to a direct measurement method performed in the field. The advantages of the method are:

1) The simultaneous but separate measurement of jolting, yaw and deformation movements

2) The simultaneous measurement of the boom speed at several points

3) The measurement of boom movements on the field 
4) The ability of implementing the measurement chain on mounted, trailed or self-propelled sprayers

5) The whole coverage of the most interesting frequency range $(0.1-12.5 \mathrm{~Hz})$

The limitations of the method are:

1) The duration of the entire test is quite high: from two hours (short track and trained people) to 12 hours ( $5 \mathrm{~km}$ of field tests with only two people).

2) The discrimination between yaw and deformation may not be absolutely complete, since the yaw $(>0.2 \mathrm{~Hz})$ is estimated on basis of a 5-point linear interpolation

3) It is not certain that the method will be applicable to any boom sprayer. If the sprayer frame is split into several parts that move independently, the correct implementation of the sensors would be difficult.

On the two trailed machines examined, the effects of the horizontal boom movements were not high in relation to those due to other causes, such as spray drift, nozzle wear and spray pressure variations. They are still significant, however. The coefficients of variation of the boom speed were between 4 and $6 \%$. The main movement was yaw, but deformation 
movements were not negligible. Jolting amplitudes were very low, except at low frequencies $(<0.2 \mathrm{~Hz})$. At these frequencies, the jolting motion is the response to the tractor motion, and is probably compensated by the modification of the pump flow rate (the latter being dependent on the wheels rotation speed, or the engine rotation speed).

The tests developed are intended to be used to compare the movements of sprayer booms in controlled conditions and to provide an understanding of their dynamic behaviour. They are currently being extended to self-propelled and mounted sprayers.

\section{Acknowledgements}

This study was funded by the S.S.T.C. (Services du Premier Ministre pour les Affaires

Scientifiques, Techniques et Culturelles. Rue de la Loi, 8, 1000 Bruxelles), project NR. $\mathrm{NM} / 01 / 06$.

\section{References}

Dagnelie, P., 1975. Théorie et méthodes statistiques. Les Presses Agronomiques de Gembloux, 1975, 107-117 
De Baerdemaker, J., Jacques, M., Verdonck, E., 1983. Modelling the Dynamic Behavior of Sprayer Booms. American Society of Agricultural Engineering, Chicago, 1983, Paper no 831507.

De Jong, A., Van de Zande, J.C., Stallinga, H., 2000. The effects of vertical and horizontal boom movements on the uniformity of spray distribution.Proc. Ag. Eng. 2000 Conference, Warwick, 2000, Paper No 00-PM-015.

Lardoux, Y., Sinfort, C., Schmidt, K., Sevila, F.,1998. Boom behaviour diagnosis from modelisation of field sprayer chemical distribution on soil. International Conference on Engineering of Decision Support Systems in Bio-industries, Bio-Decision 98, Montpellier, France.

Lebeau, F., Destain, M.-F., 1998. Measurement of the Sprayer Boom Displacements with a Laser Sensor Displacement. Proc. Ag. Eng. 1998 Conference, Oslo, 1998, Paper No 98-A004.

Nation, H.J., 1982. The Dynamic Behaviour of Field Sprayer Booms. Journal of Agricultural Engineering Research. (1982) 27, 61-70. 
Sinfort, C., Lardoux, Y., Miralles, A., Enfalt, P., Alness, K., Andersson, S., 1997. Comparison between measurements and predictions of spray pattern from a moving boom sprayer. Aspects of Applied Biology 48, 8 p.

Speelman, L., Jansen, J.W., 1974. The effect of Spray-boom Movement on the Liquid Distribution of Field Crop Sprayers. Journal of Agricultural Engineering Research (1974) 19, 117-129.

Vannucci, D., Pari, L., Fedrizzi, M., Menesatti, P., 1992. Study of an automatic system to determine the spraying boom lateral fluctuation. Proc. Ag. Eng. Conference, UppsalaSweden, 1992., paper No 9205113, 5 p. 


\section{Figure captions}

Fig. 1. Different types of spraying machines: (a) mounted (b) trailed (c) self-propelled.

Fig. 2. Schema of a trailed sprayer machine. Yaw and jolting motions of the boom.

Fig. 3. The steps in the movements measurement and calculation.

Fig. 4. Disposition of the sensors on a trailed spraying machine.

Fig. 5. Testing for study of the dynamic behaviour of the boom sensors.

Fig. 6. Dynamic performance of the boom sensors.

Fig. 7. Determination of the cut-off frequency of the high-pass filter to stay within $5 \%$ error in the measured speed signal.

Fig. 8. The data acquisition system.

Fig. 9a. Data preprocessing and processing: boom movements at high frequencies $(>0.2 \mathrm{~Hz})$.

Fig. 9b. Data preprocessing and processing: relative movements between the boom and the frame at low frequencies $(<0.2 \mathrm{~Hz})$.

Fig. 9c. Data preprocessing and processing: movements of the frame at low frequencies $(<0.2$ $\mathrm{Hz})$.

Fig. 10. Sensor fusion. 
Fig. 11. Comparison with a laser measurement method: disposition of the laser sensor.

Fig. 12. Comparison of the sensor fusion method and the laser measurement: mounted sprayer with $18 \mathrm{~m}$ boom length at $5.6 \mathrm{~km} / \mathrm{h}$ on fallow.

Fig. 13. Yaw spectra of sprayer 1.

Fig. 14. Jolting spectra of sprayer 1.

Fig. 15. Deformation spectra of sprayer 1.

Fig. 16. Yaw spectra of sprayer 2.

Fig. 17. Deformation spectra of sprayer 2. 

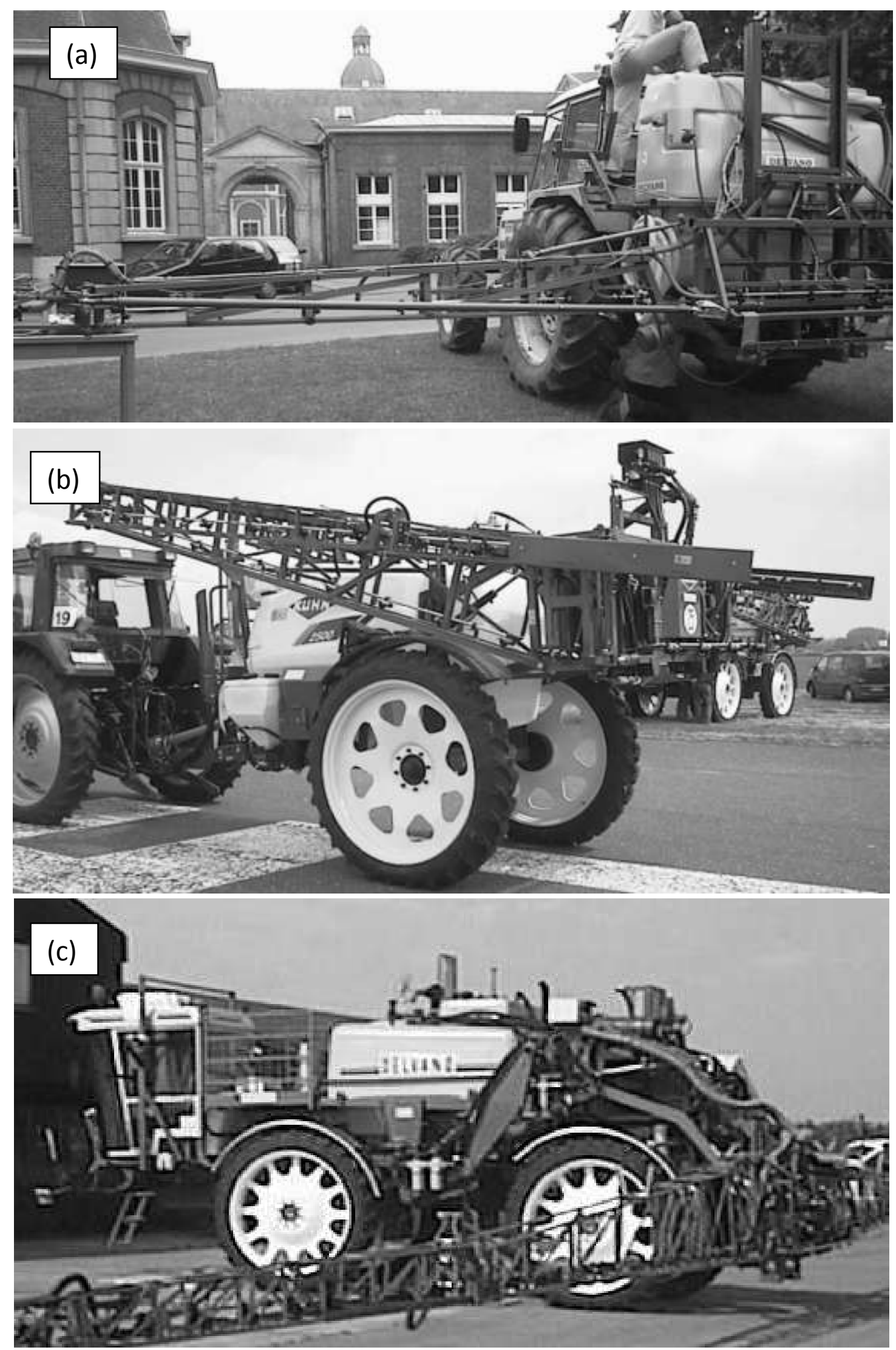

Fig. 1. 


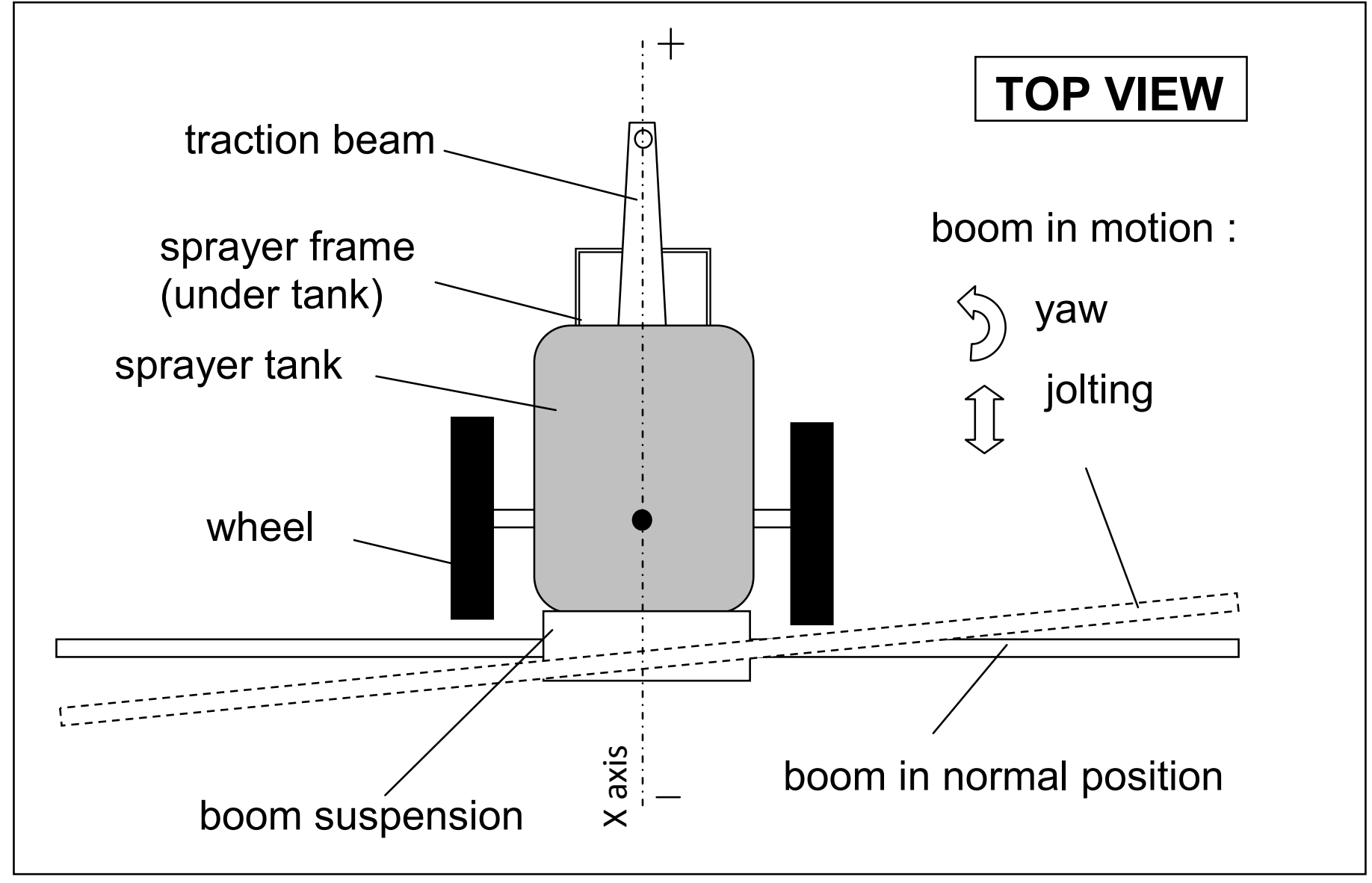

Fig. 2 


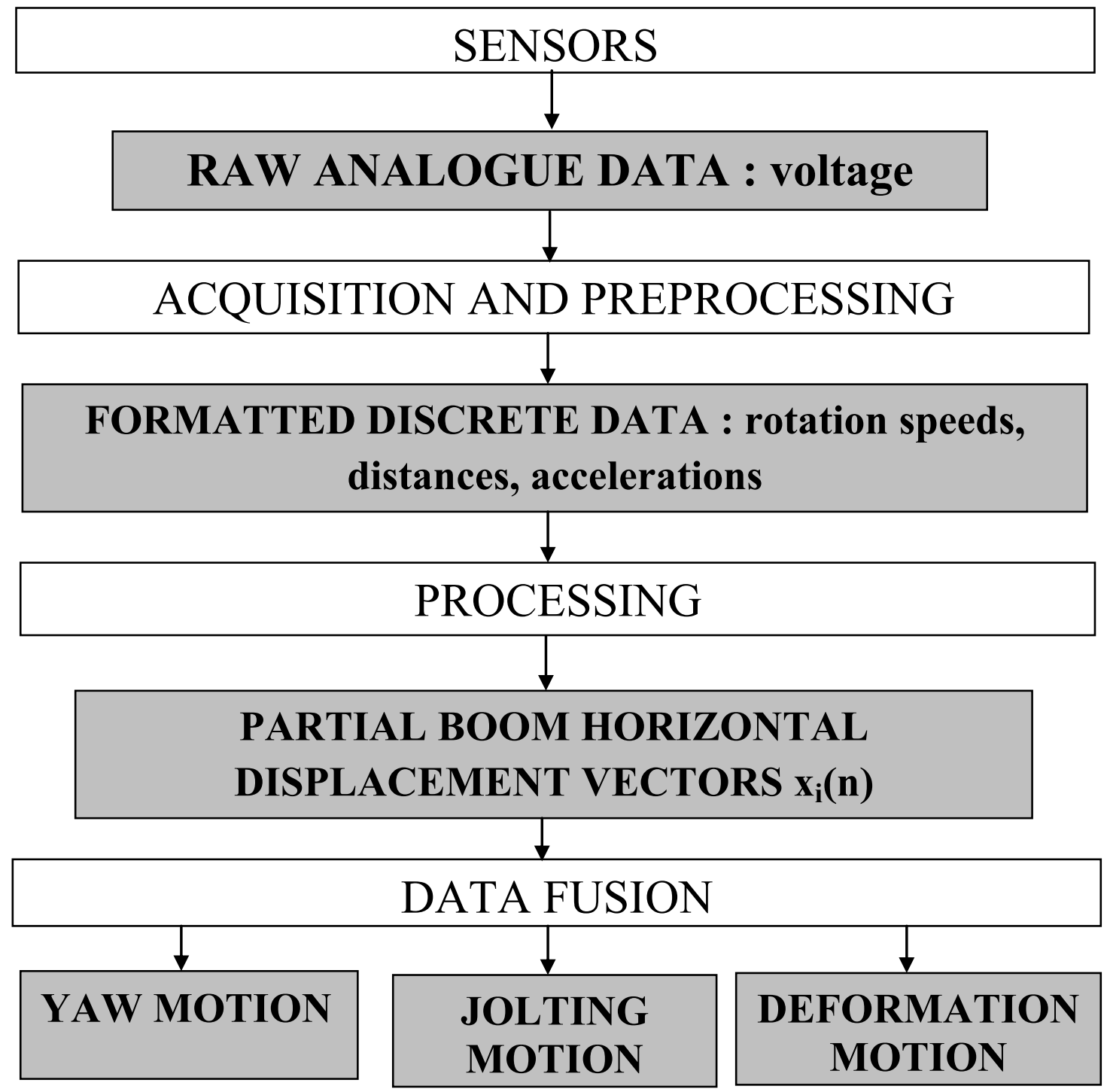

Fig. 3. 


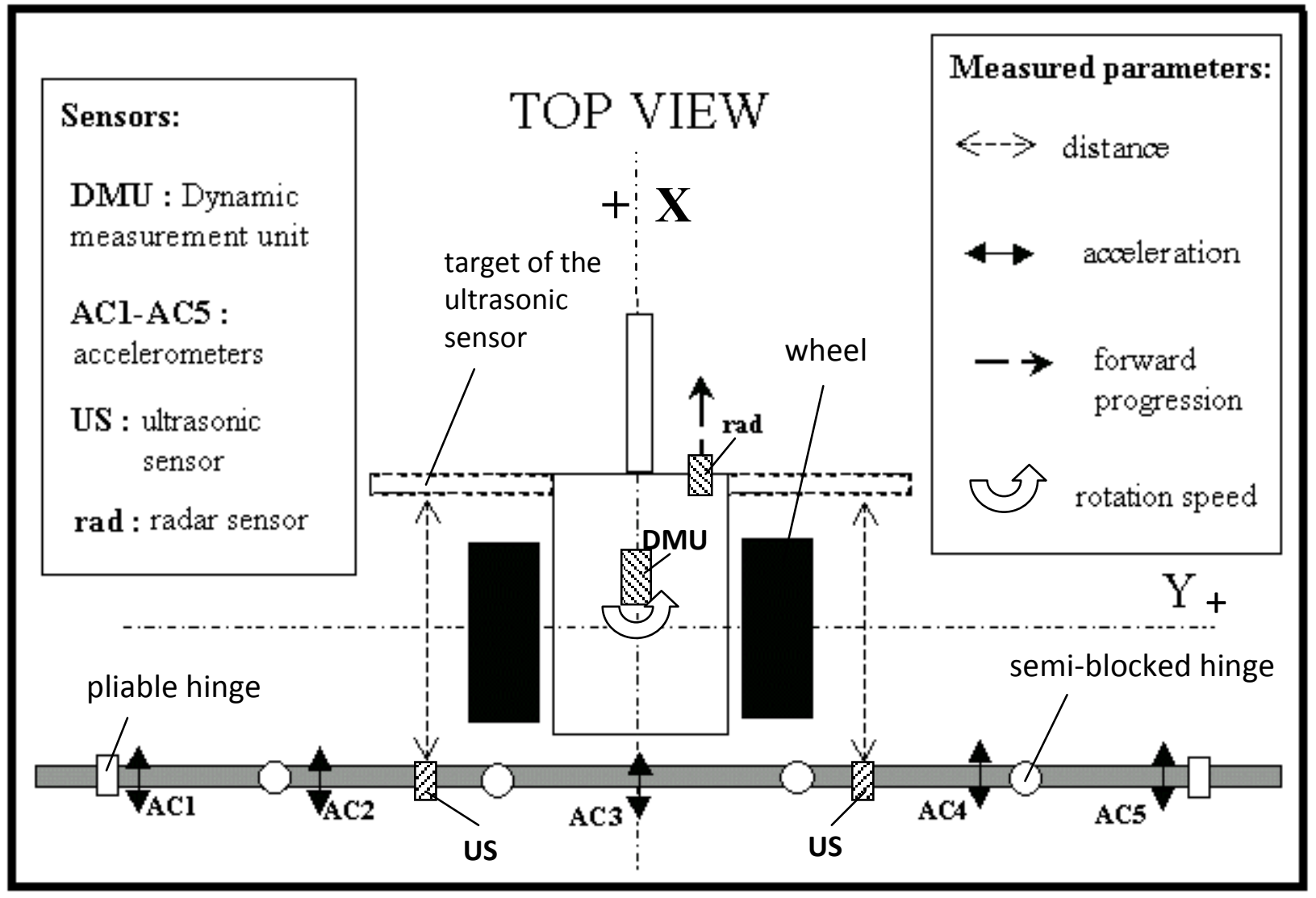

Fig. 4 


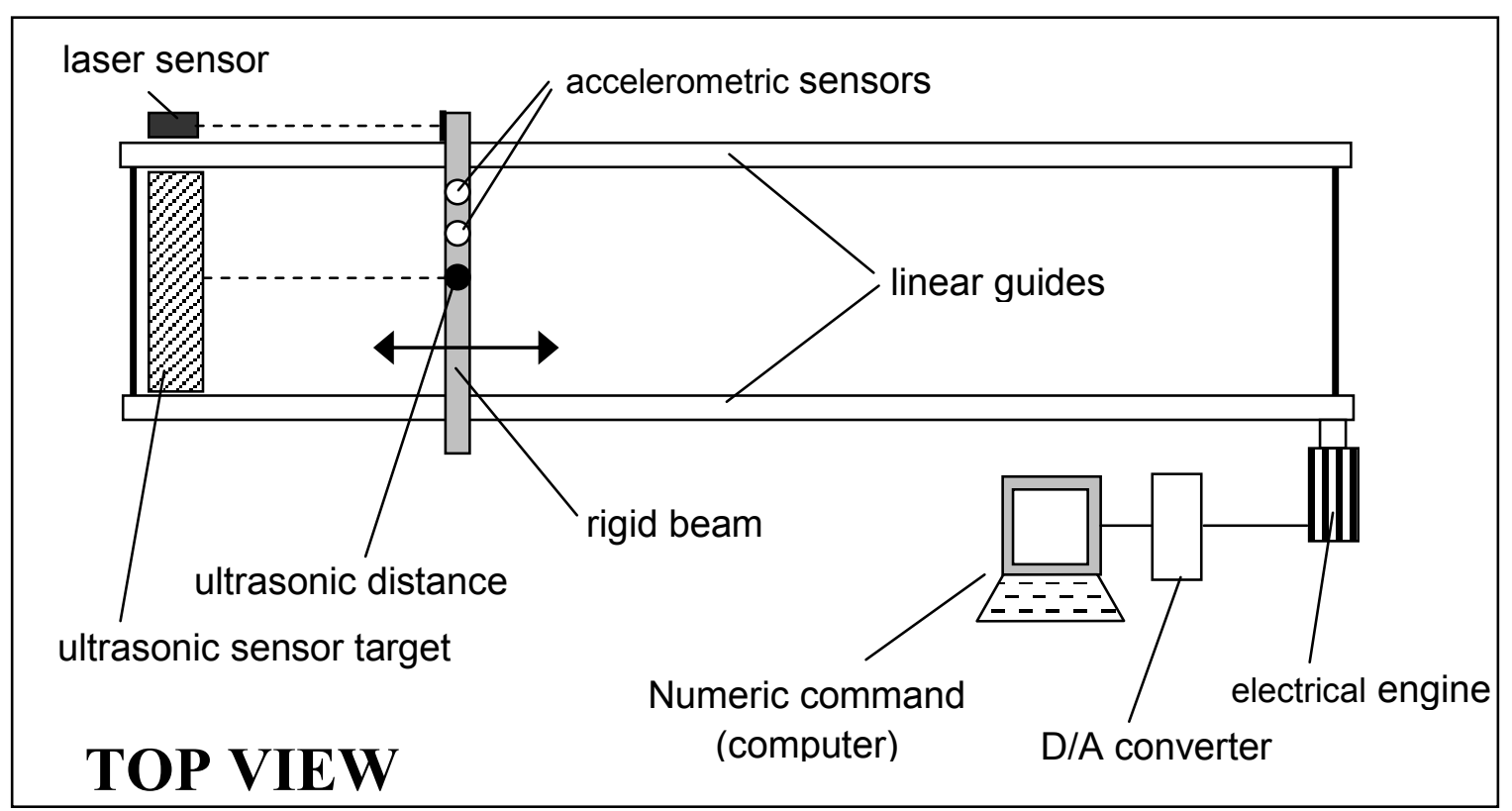

Fig. 5 


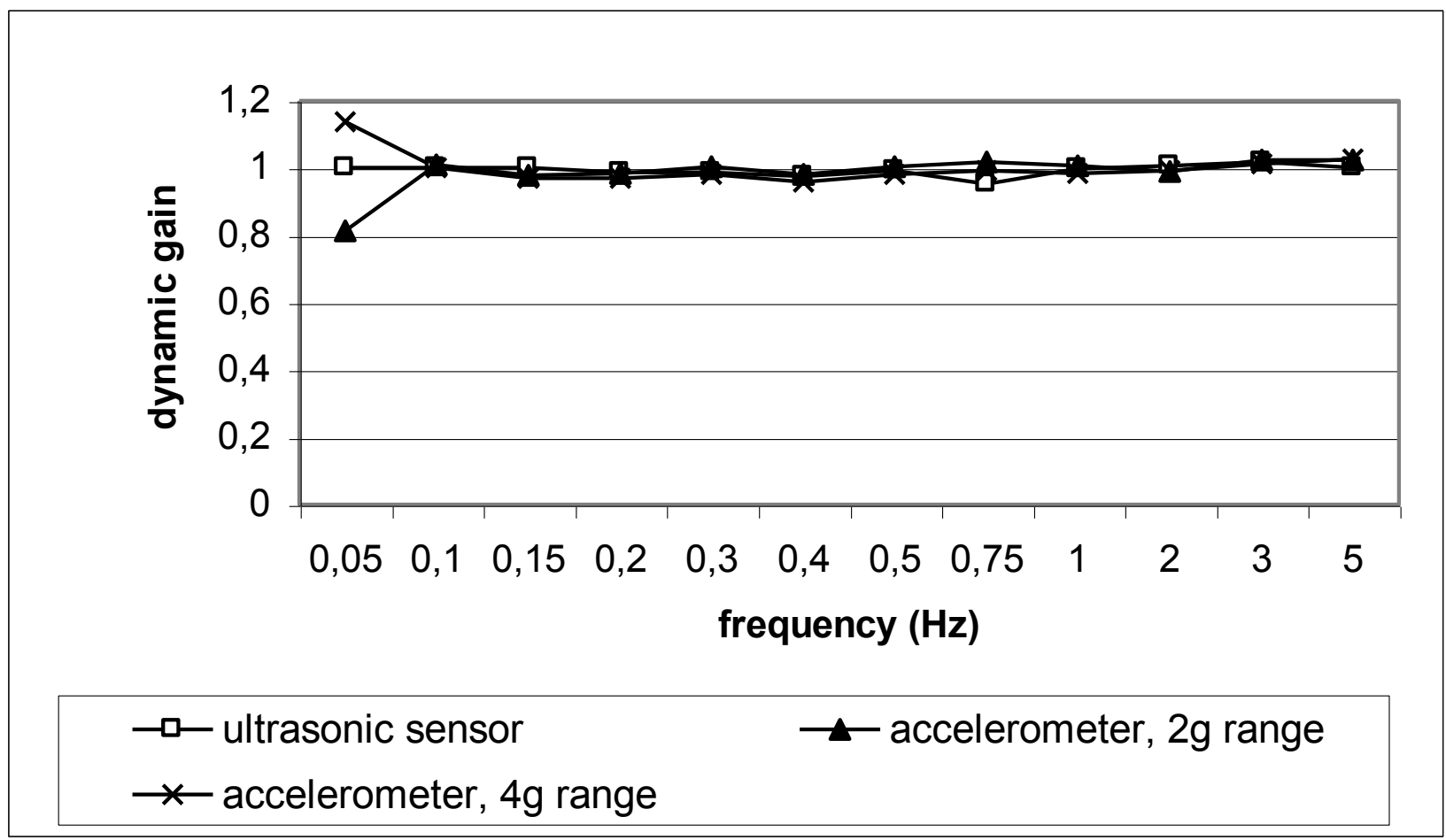

Fig. 6 


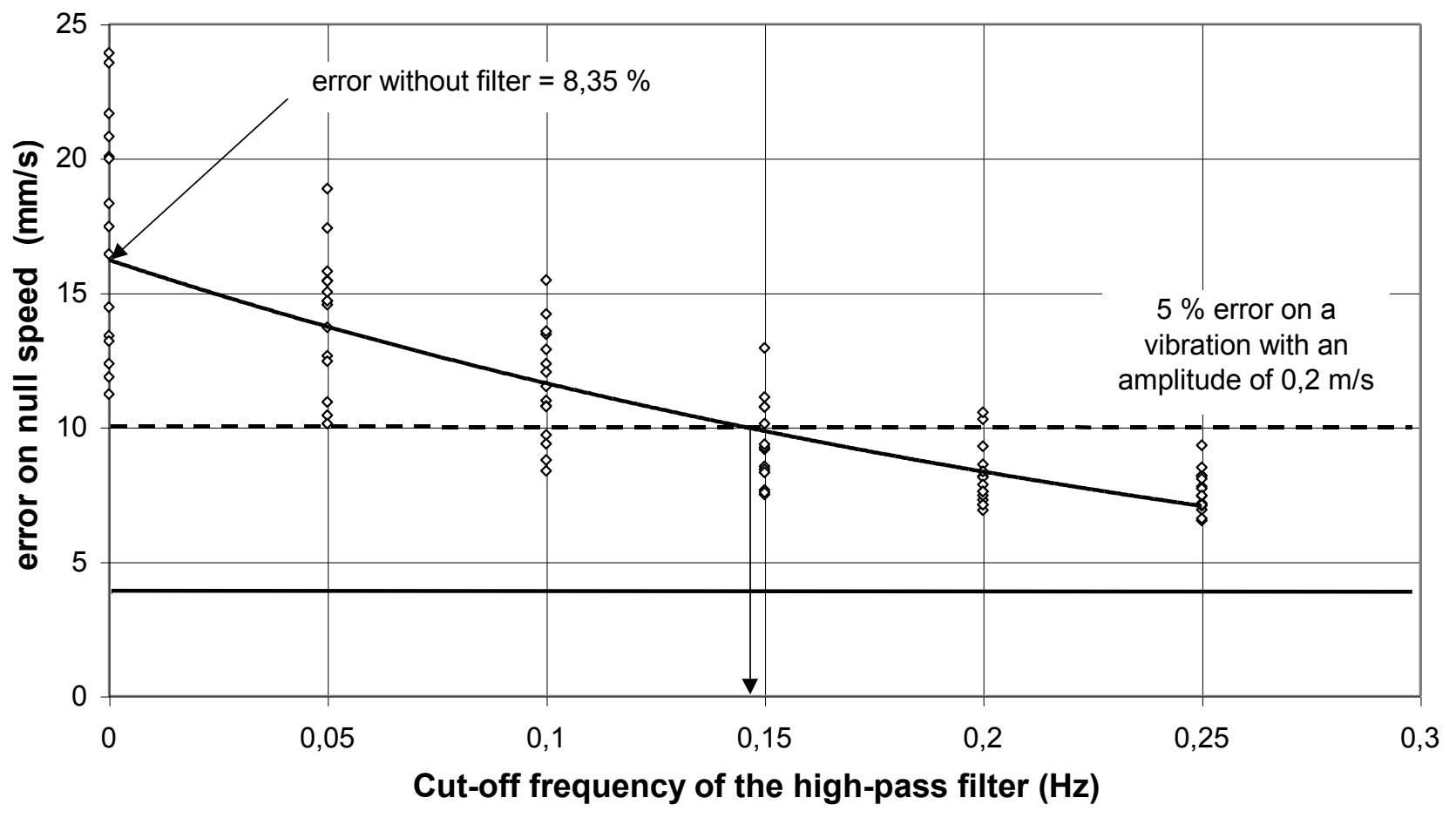

Fig. 7 


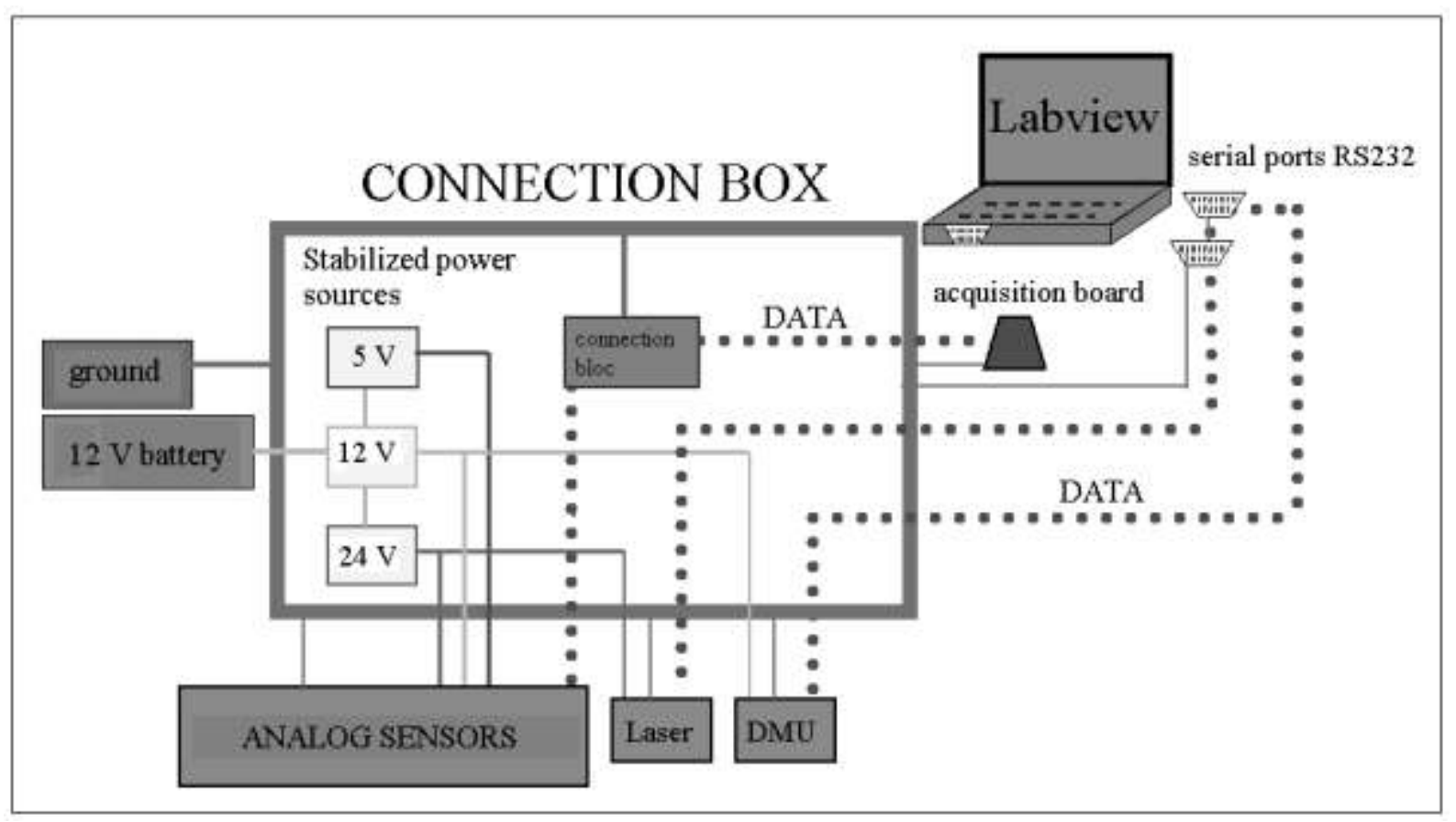

Fig. 8 


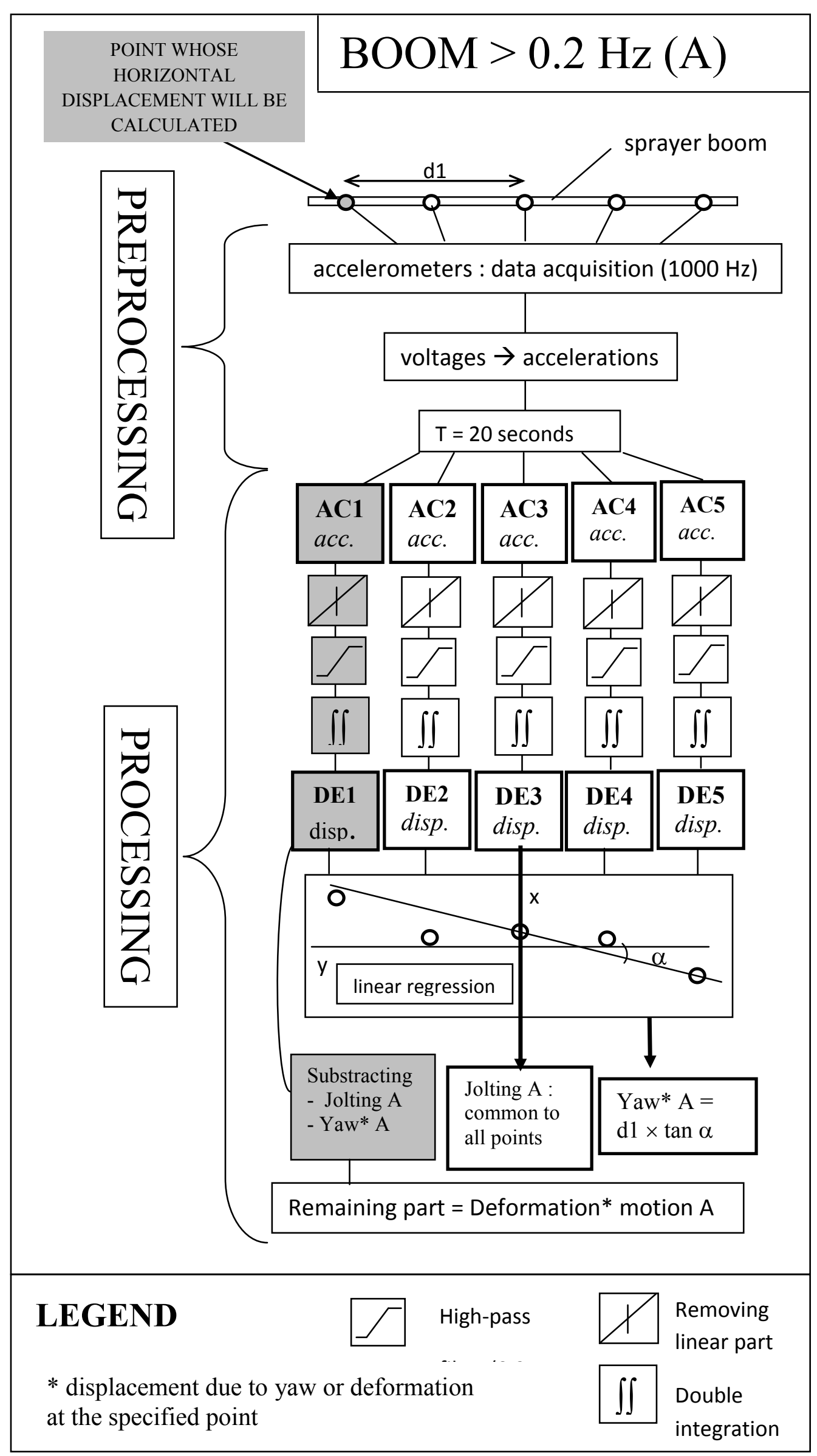

Fig. 9 


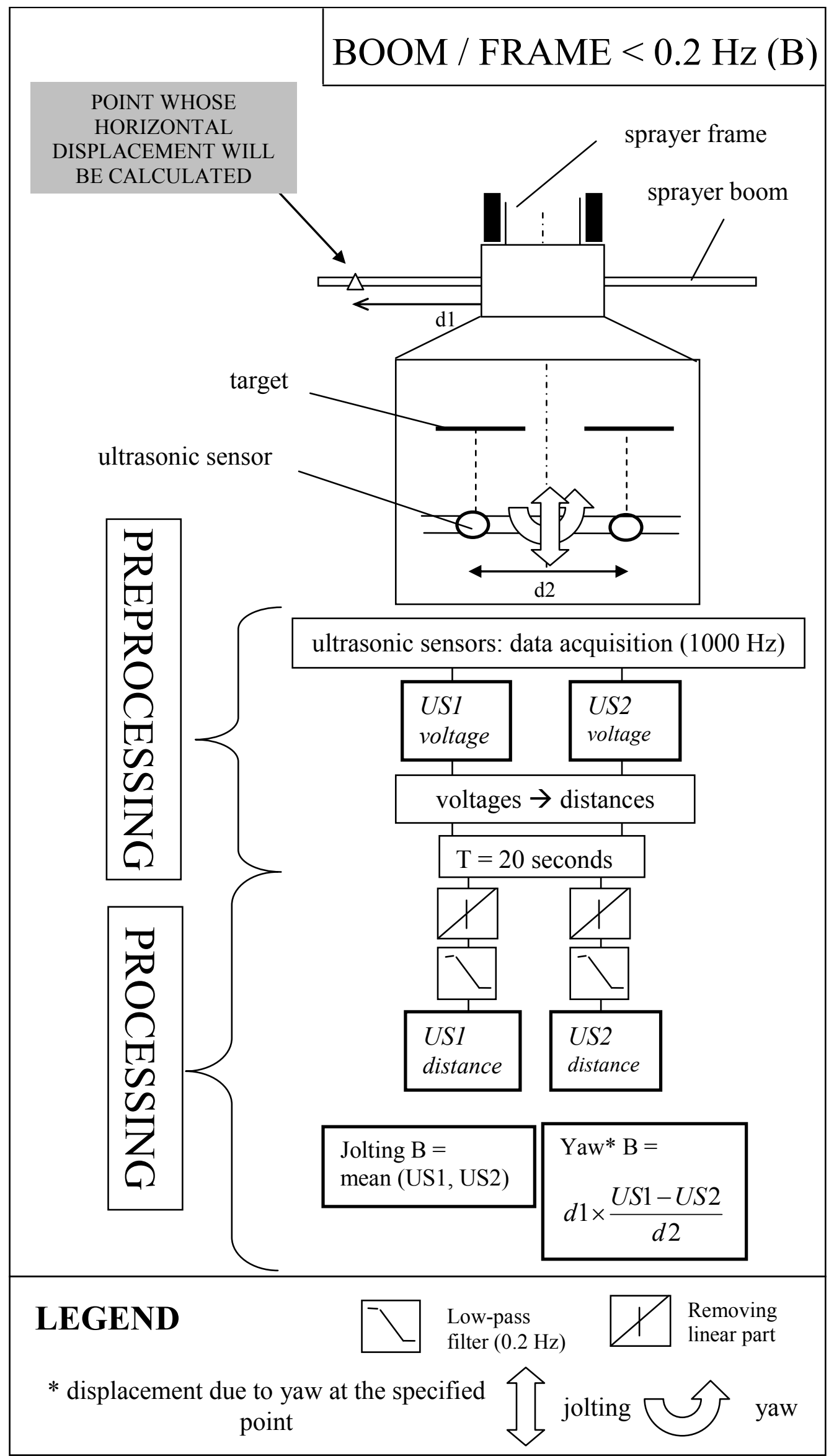




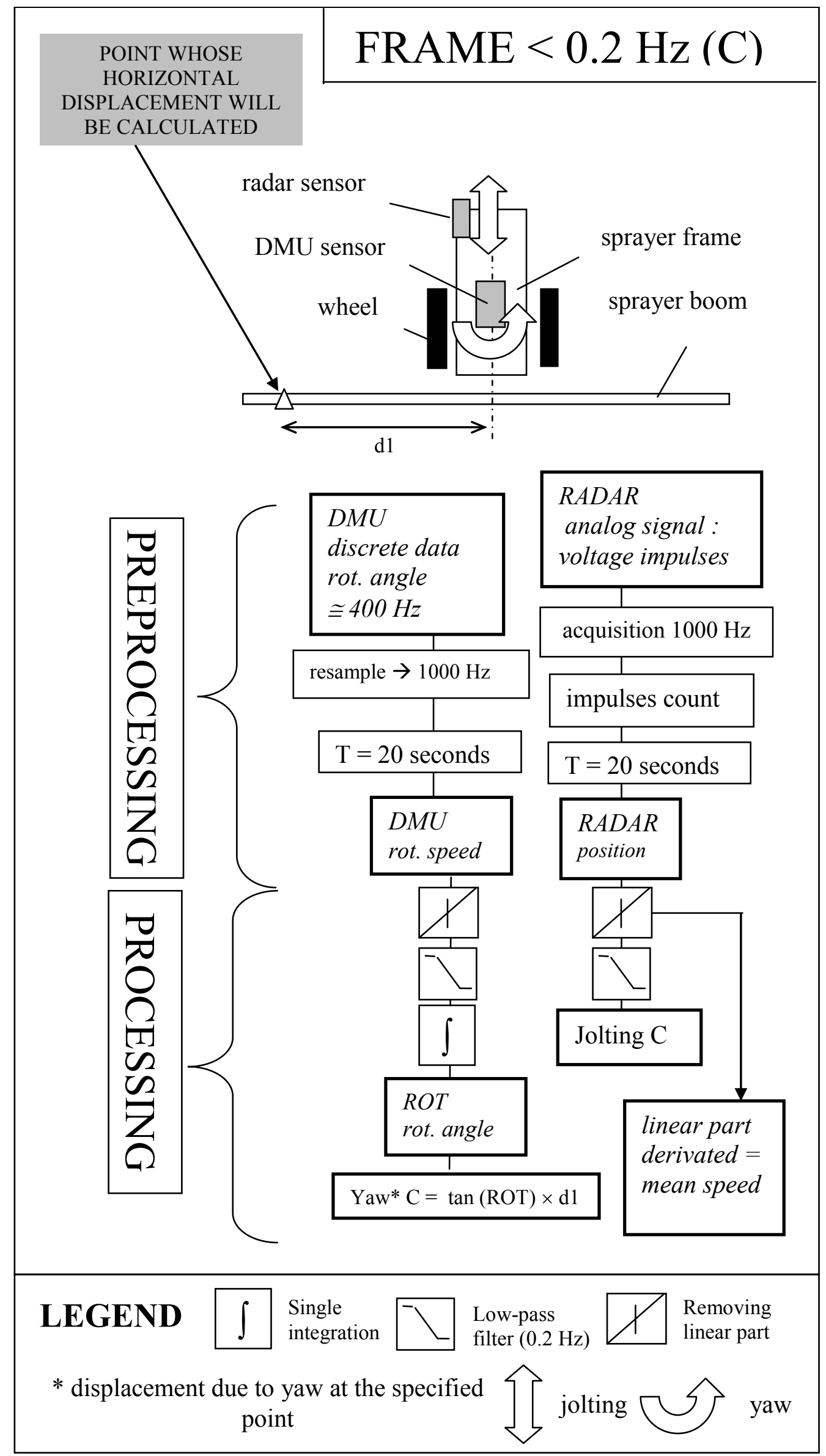




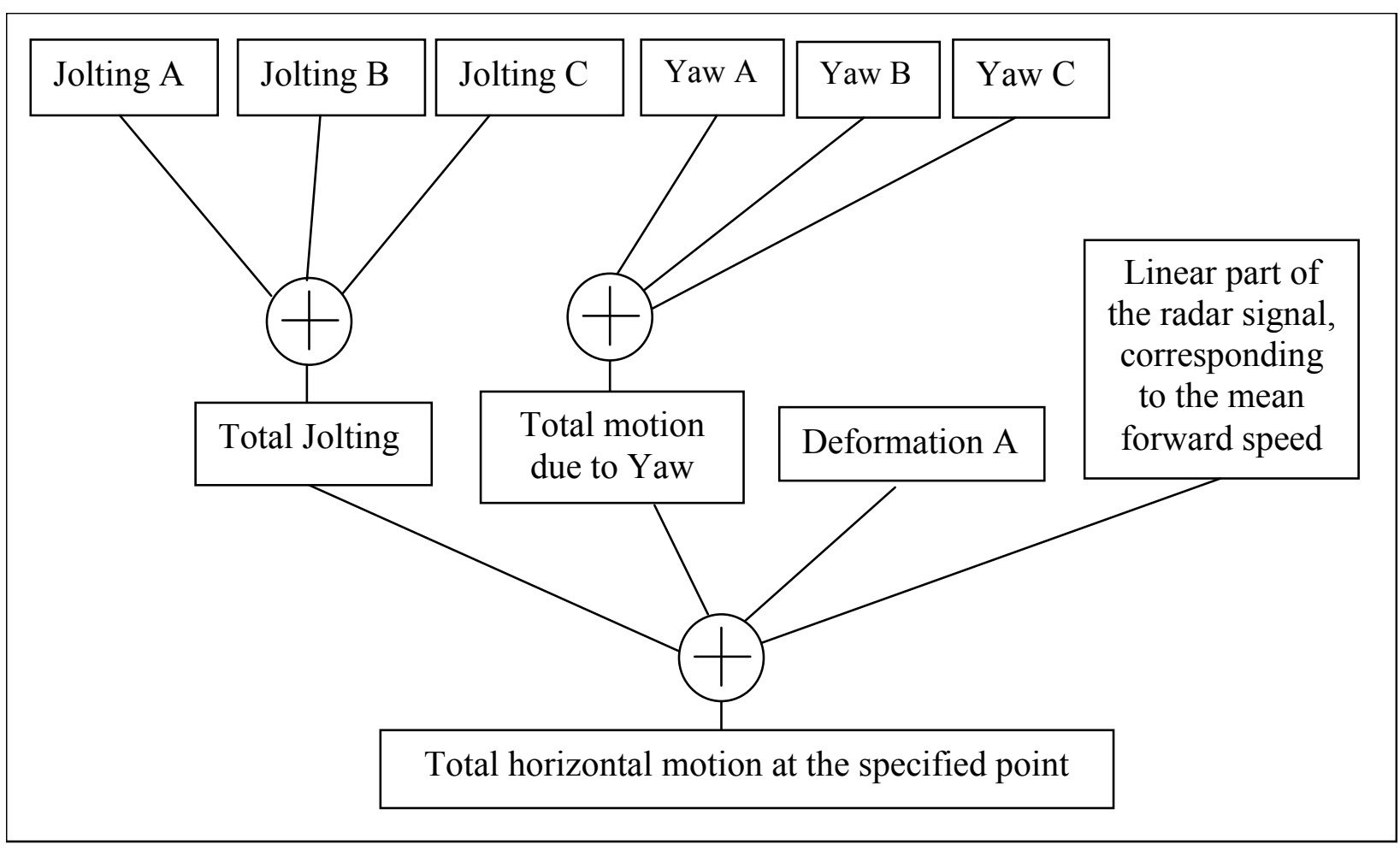

Fig. 10 


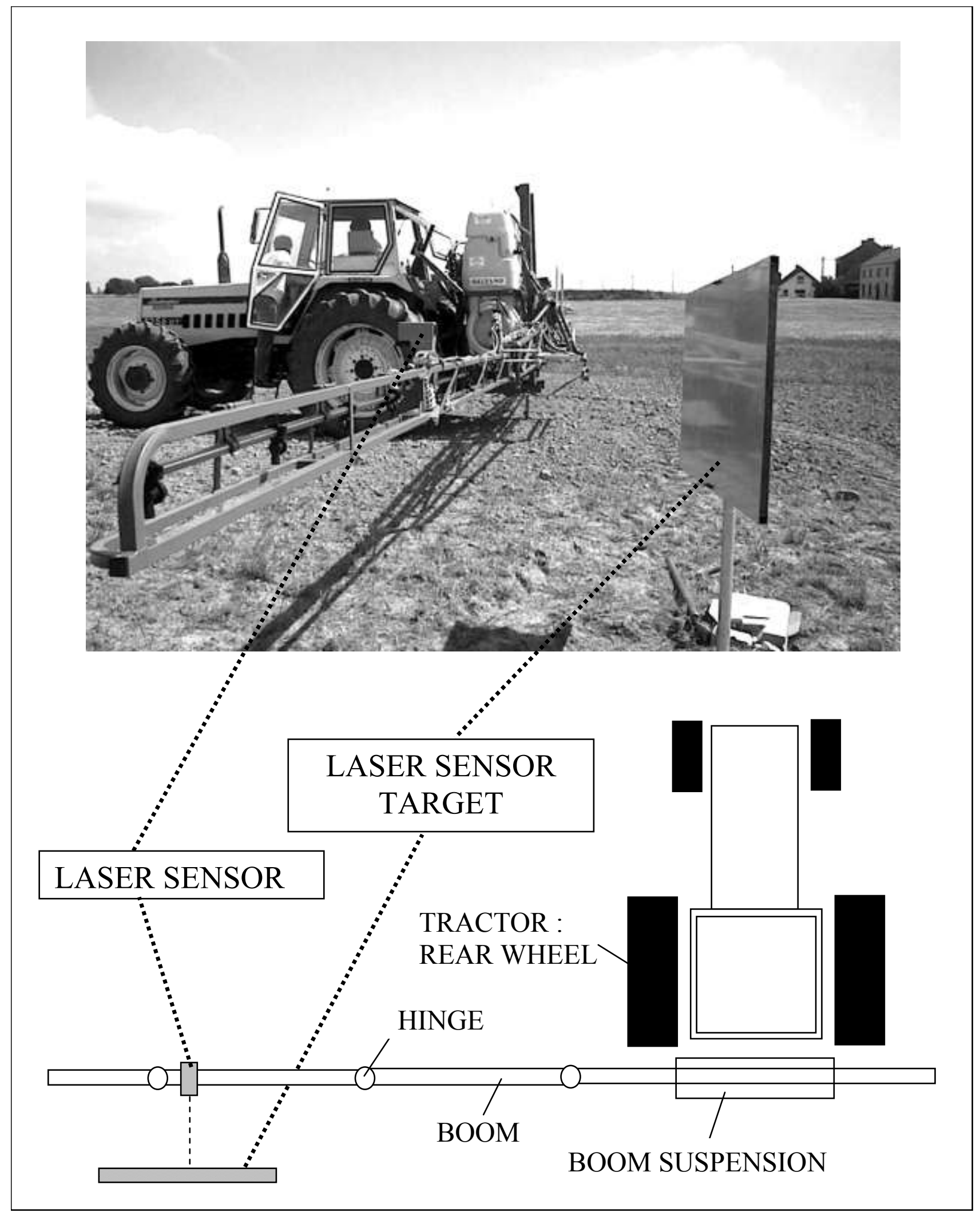

Fig. 11 


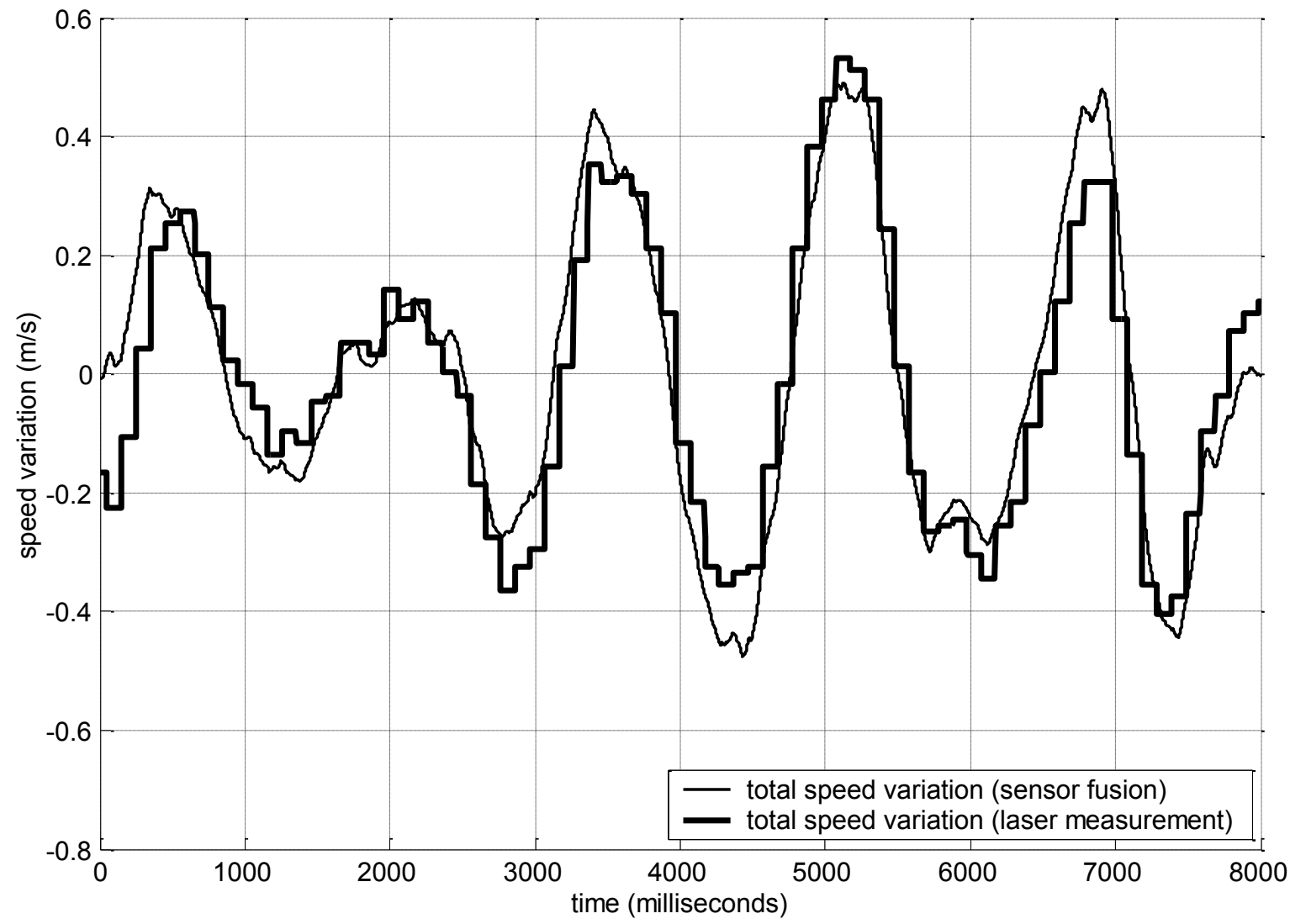

Fig. 12 


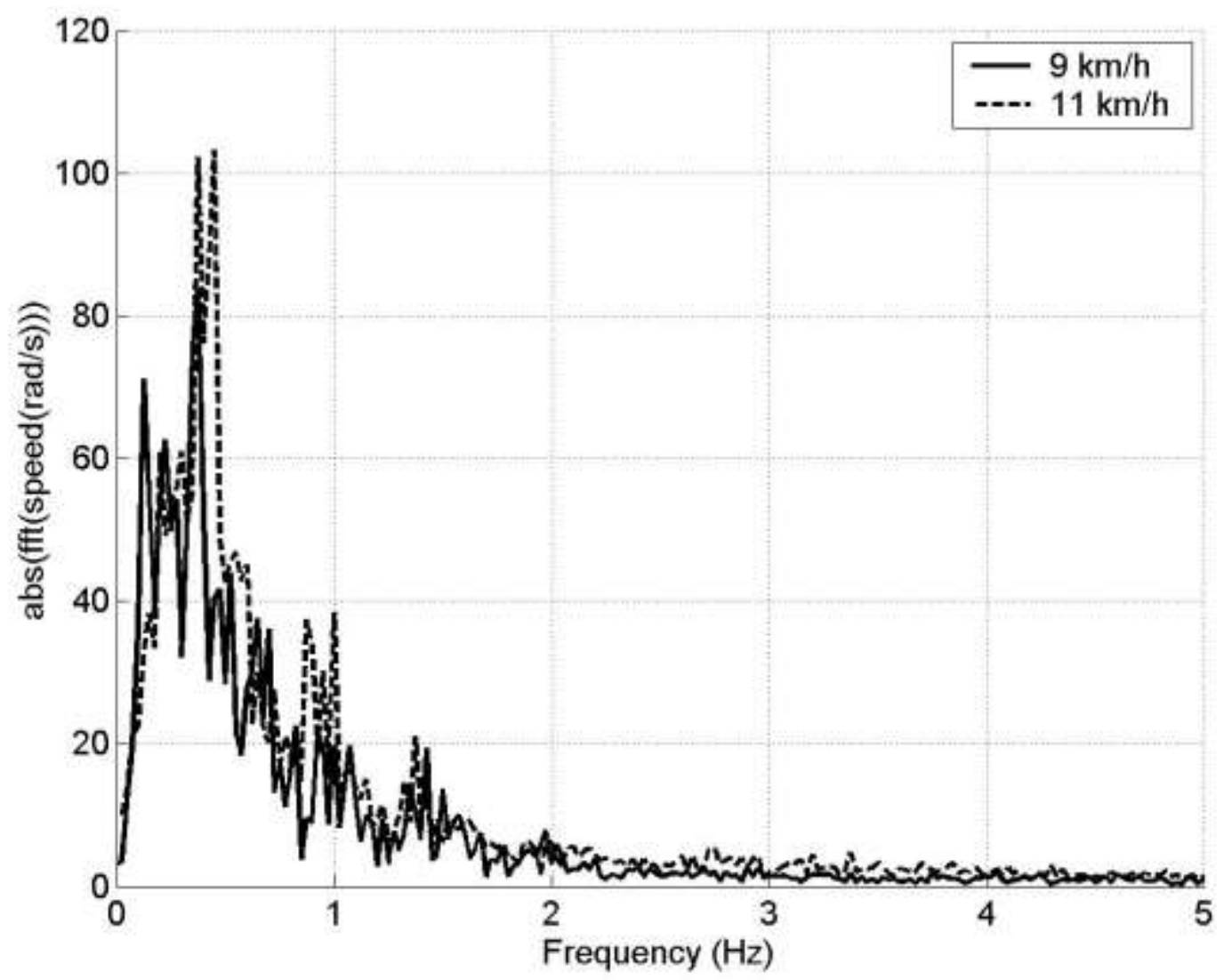

Fig. 13 


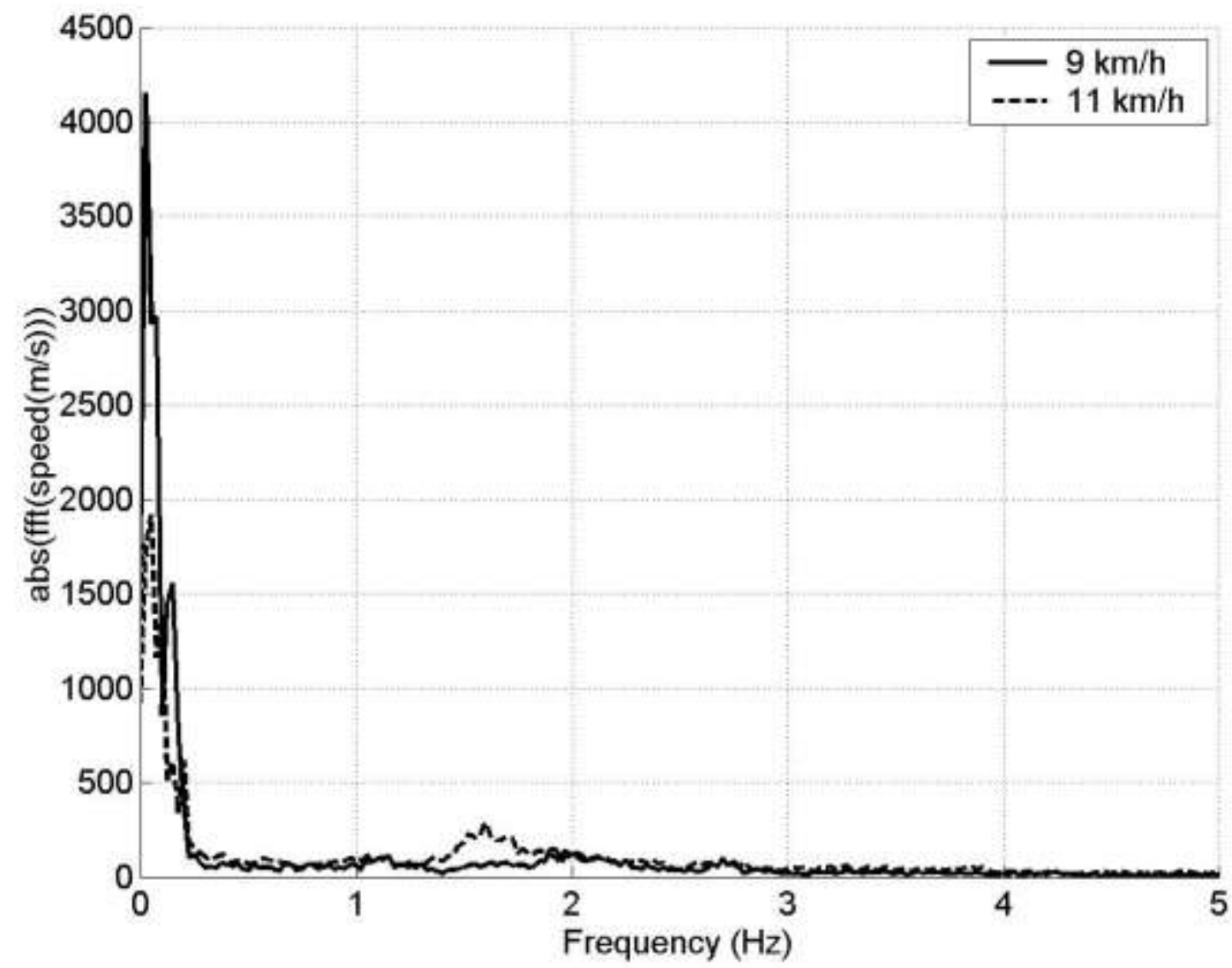

Fig. 14 


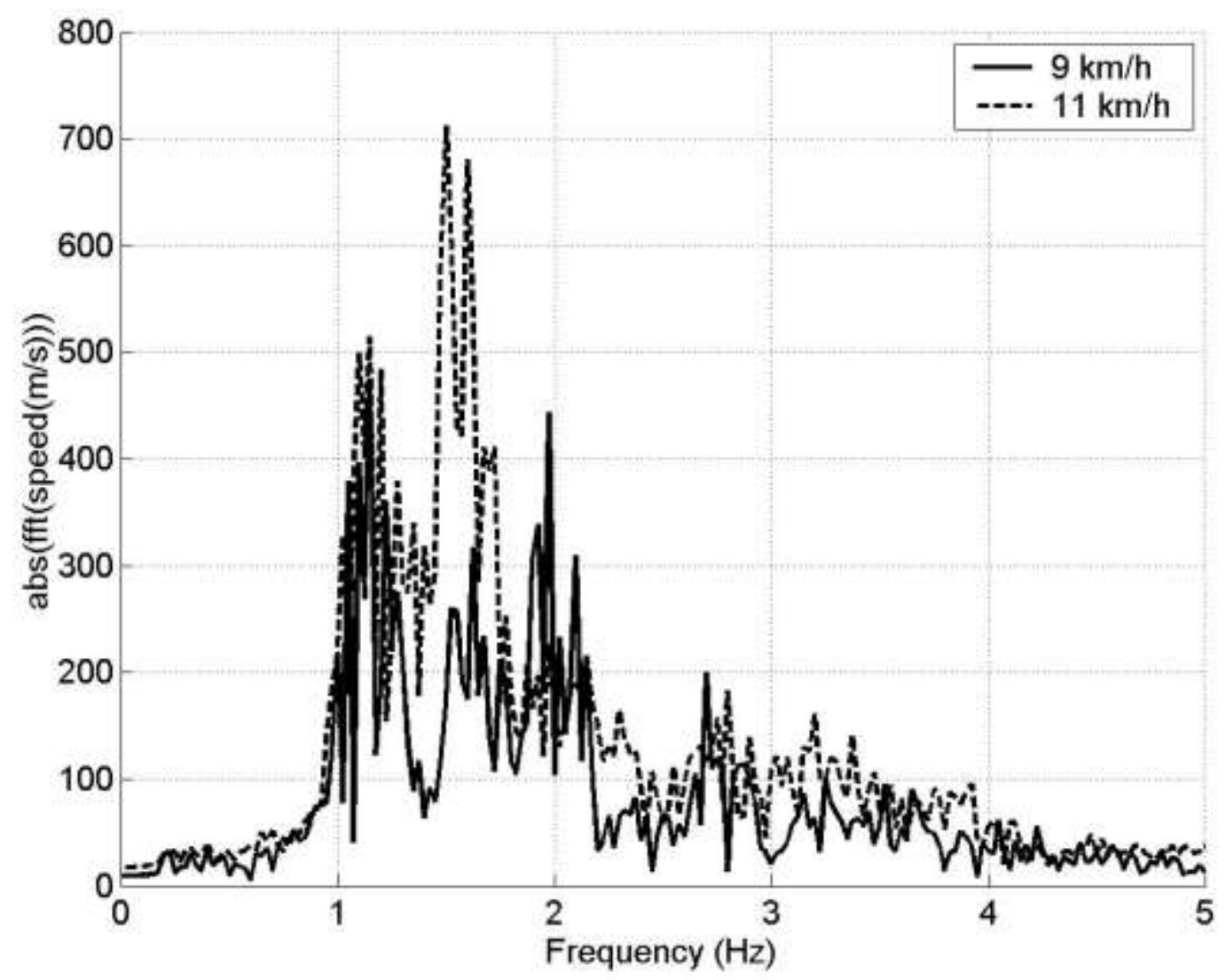

Fig. 15 


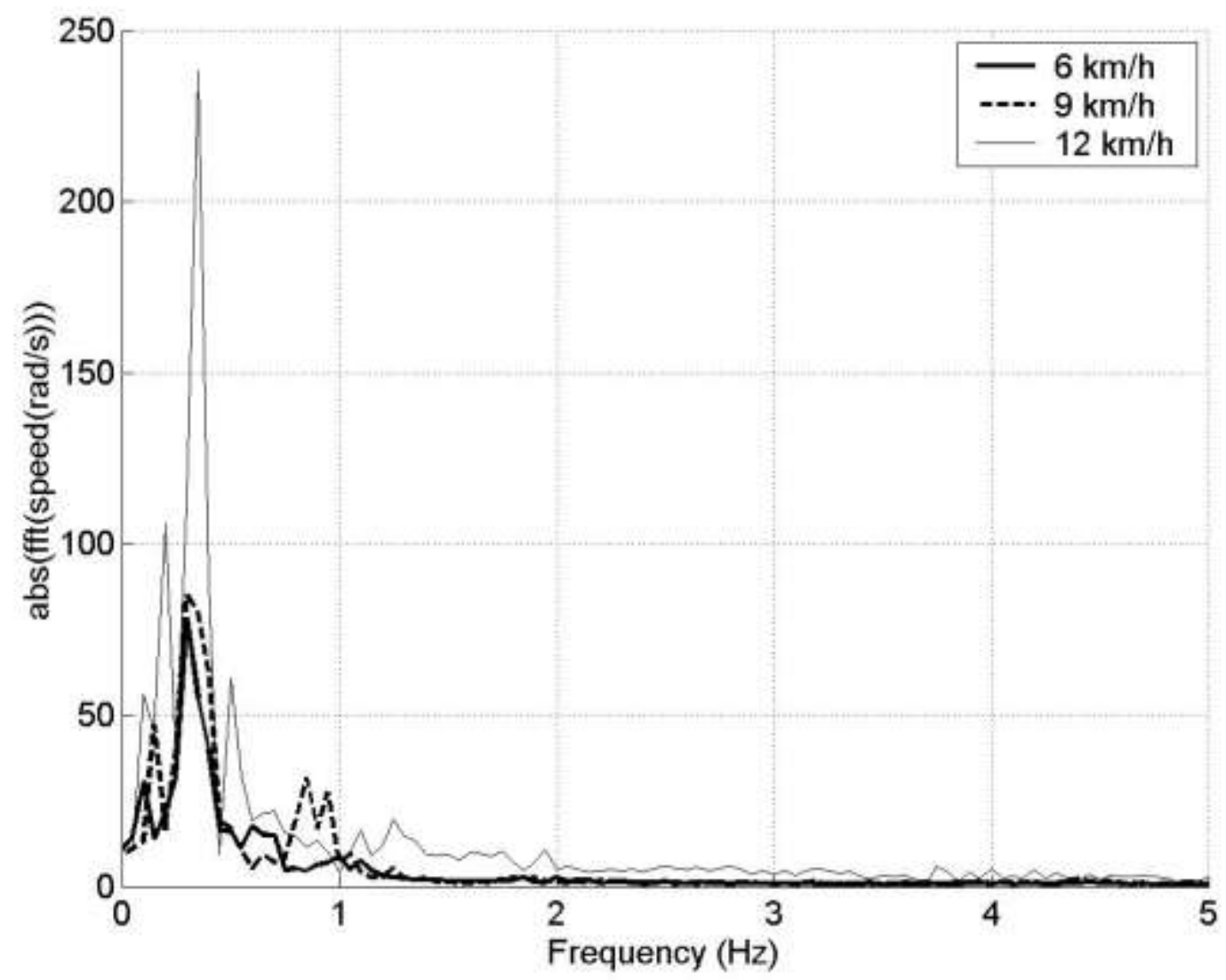

Fig. 16 


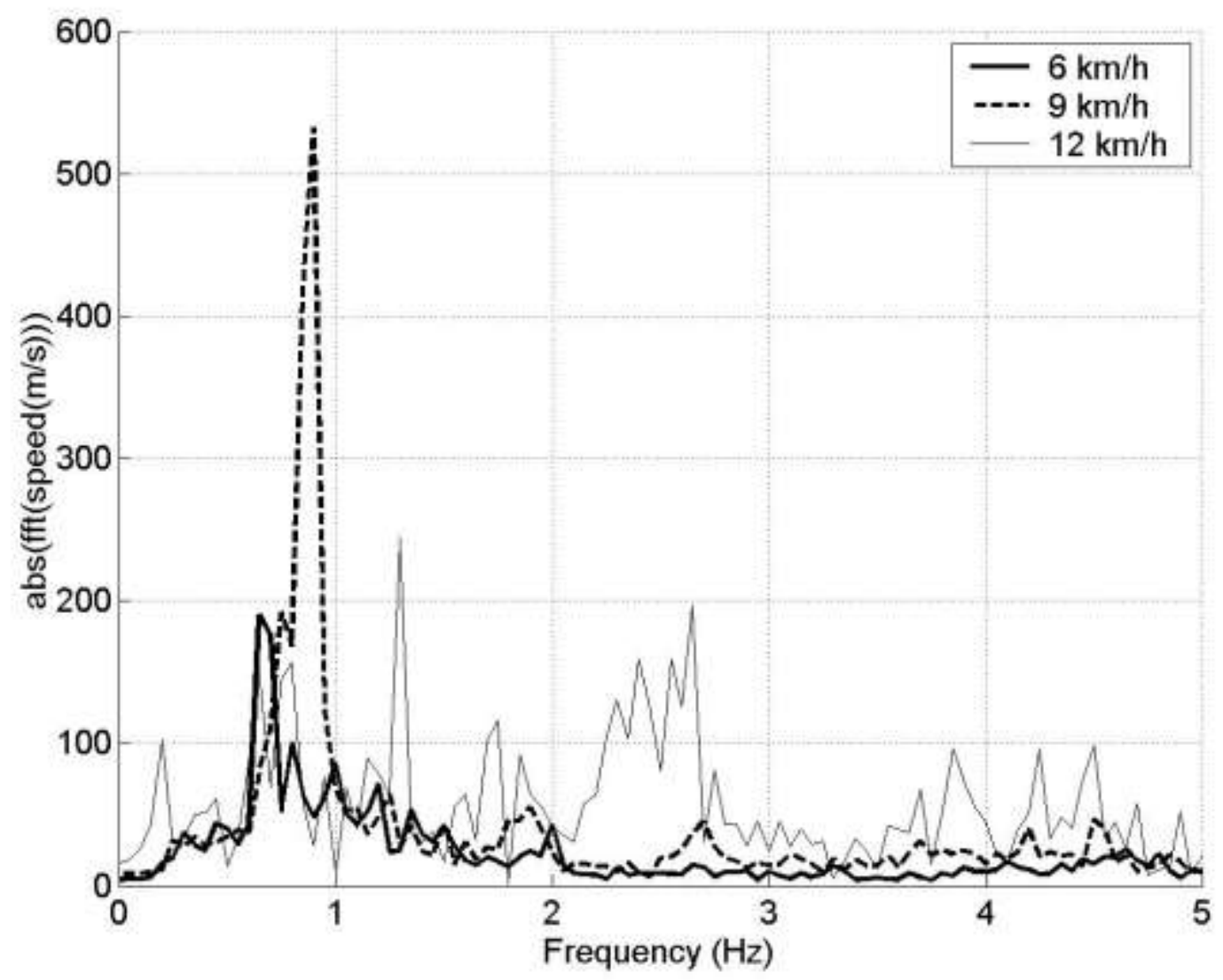

Fig. 17 
Table 1. Recapitulative of trials.

\begin{tabular}{ccc}
\hline Machine & \multicolumn{1}{c}{1} & \multicolumn{1}{c}{2} \\
& $\begin{array}{c}\text { - Trailed } \\
\text { - boom length }=22 \mathrm{~m} \\
\text { - Empty tank }\end{array}$ & $\begin{array}{l}\text { - Trailed } \\
\text { - boom length }=24 \mathrm{~m} \\
\text { - } 1 / 10 \text { full tank }\end{array}$ \\
\hline Date & 18,19 th July,2000 & 1 2th October,2000 \\
\hline Place & Gembloux, Belgium & Merelbeke, Belgium \\
\hline Soil & Dry and very compact & Wet \\
\hline Crop & Wheat & Meadow \\
\hline Speed & 9 and $11 \mathrm{~km} / \mathrm{h}$ & 6,9 and $12 \mathrm{~km} / \mathrm{h}$ \\
\hline
\end{tabular}


Table 2: Horizontal vibrations frequencies of the sprayer booms at $8,5 \mathrm{~m}$ from the centre

\begin{tabular}{cccccccc}
\hline Sprayer & Tank & Speed & Field & ${ }^{*}$ & Yaw & Jolting & Deformation \\
\hline 1 & \multirow{2}{*}{ Empty } & \multirow{2}{*}{$9 \mathrm{~km} / \mathrm{h}$} & \multirow{2}{*}{ wheat } & $\begin{array}{c}\mathrm{M} \\
\mathrm{S}\end{array}$ & $0,35 \mathrm{~Hz}$ & $2 \mathrm{~Hz}$ & 1 to $2 \mathrm{~Hz}$ \\
\hline 1 & \multirow{2}{*}{ Empty } & \multirow{2}{*}{$11 \mathrm{~km} / \mathrm{h}$} & \multirow{2}{*}{ wheat } & $\begin{array}{c}\mathrm{M} \\
\mathrm{S}\end{array}$ & $0,35 \mathrm{~Hz}$ & $1,65 \mathrm{~Hz}$ & $1 \mathrm{to} 2 \mathrm{~Hz}$ \\
\hline 2 & \multirow{2}{*}{$1 / 10$ full } & \multirow{2}{*}{$6 \mathrm{~km} / \mathrm{h}$} & \multirow{2}{*}{ meadow } & $\begin{array}{c}\mathrm{M} \\
\mathrm{S}\end{array}$ & $0,325 \mathrm{~Hz}$ & $?$ & $0,65 \mathrm{~Hz}$ \\
& \multirow{2}{*}{$1 / 10$ full } & \multirow{2}{*}{$9 \mathrm{~km} / \mathrm{h}$} & \multirow{2}{*}{ meadow } & $\begin{array}{c}\mathrm{M} \\
\mathrm{S}\end{array}$ & $0,325 \mathrm{~Hz}$ & $?$ & $0,9 \mathrm{~Hz}$ \\
\hline 2 & \multirow{2}{*}{$1 / 10$ full } & \multirow{2}{*}{$12 \mathrm{~km} / \mathrm{h}$} & \multirow{2}{*}{ meadow } & $\begin{array}{c}\mathrm{M} \\
\mathrm{S}\end{array}$ & $0,35 \mathrm{~Hz}$ & $?$ & $1,25 \mathrm{~Hz}$ \\
\end{tabular}

${ }^{*} \mathrm{M}=$ Main mode, $\mathrm{S}=$ Secundary mode, ? = unknown 
Table 3: Horizontal velocities of the sprayer booms at 8,5 $\mathrm{m}$ from the center

\begin{tabular}{|c|c|c|c|c|c|c|c|c|c|}
\hline Sprayer & Tank & Speed & Field & * & Yaw & Deform. & $\begin{array}{r}0,2 \mathrm{~Hz} \\
\text { Jolting } \\
\end{array}$ & Total & units \\
\hline & & & & $\bar{V}$ & 0,0071 & 0,0044 & 0,00081 & 0,011 & $\mathrm{~m}^{2} / \mathrm{s}^{2}$ \\
\hline \multirow[t]{3}{*}{1} & Empty & $9 \mathrm{Km} / \mathrm{h}$ & 2 & $\mathrm{CV}$ & 3,6 & 2,8 & 1,2 & 4,4 & $\%$ \\
\hline & & & & $\mathrm{M}$ & 0,24 & 0,24 & 0,11 & 0,37 & $\mathrm{~m} / \mathrm{s}$ \\
\hline & & & & V & 0,013 & 0,011 & 0,0017 & 0,022 & $\mathrm{~m}^{2} / \mathrm{s}^{2}$ \\
\hline \multirow[t]{3}{*}{1} & Empty & $11 \mathrm{~km} / \mathrm{h}$ & 2 & $\mathrm{CV}$ & 4,0 & 3,7 & 1,4 & 5,1 & $\%$ \\
\hline & & & & $M$ & 0,31 & 0,36 & 0,13 & 0,44 & $\mathrm{~m} / \mathrm{s}$ \\
\hline & & & & $\mathrm{V}$ & 0,0066 & 0,00081 & 0,00018 & 0,0072 & $\mathrm{~m}^{2} / \mathrm{s}^{2}$ \\
\hline \multirow[t]{3}{*}{2} & $1 / 10$ full & $6 \mathrm{~km} / \mathrm{h}$ & 3 & $\mathrm{CV}$ & 4,7 & 1,7 & 0,8 & 4,9 & $\%$ \\
\hline & & & & M & 0,21 & 0,09 & 0,05 & 0,24 & $\mathrm{~m} / \mathrm{s}$ \\
\hline & & & & $\mathrm{V}$ & 0,011 & 0,0034 & 0,00026 & 0,014 & $\mathrm{~m}^{2} / \mathrm{s}^{2}$ \\
\hline \multirow[t]{3}{*}{2} & $1 / 10$ full & $9 \mathrm{~km} / \mathrm{h}$ & 3 & $\mathrm{CV}$ & 4,1 & 2,3 & 0,6 & 4,7 & $\%$ \\
\hline & & & & $M$ & 0,23 & 0,16 & 0,06 & 0,31 & $\mathrm{~m} / \mathrm{s}$ \\
\hline & & & & $\mathrm{V}$ & 0,041 & 0,0028 & 0,00056 & 0,041 & $\mathrm{~m}^{2} / \mathrm{s}^{2}$ \\
\hline \multirow[t]{2}{*}{2} & $1 / 10$ full & $12 \mathrm{~km} / \mathrm{h}$ & 3 & $\mathrm{CV}$ & 5,7 & 1,5 & 0,7 & 5,7 & $\%$ \\
\hline & & & & $\mathrm{M}$ & 0,45 & 0,20 & 0,08 & 0,64 & $\mathrm{~m} / \mathrm{s}$ \\
\hline
\end{tabular}

${ }^{*} \mathrm{~V}, \mathrm{CV}, \mathrm{M}$ : speed variance, coefficient of variation and maximum amplitude (mean of all trials) 
Table 4. Coefficients of variation on the ground deposit due to horizontal boom motions at $8,5 \mathrm{~m}$ right to the center of the boom

\begin{tabular}{rcc}
\hline Sprayer & Speed & CV \\
\hline 1 & $9 \mathrm{~km} / \mathrm{h}$ & $4,5 \%$ \\
\hline 1 & $11 \mathrm{~km} / \mathrm{h}$ & $5,1 \%$ \\
\hline 2 & $6 \mathrm{~km} / \mathrm{h}$ & $5,1 \%$ \\
\hline 2 & $9 \mathrm{~km} / \mathrm{h}$ & $4,4 \%$ \\
\hline 2 & $12 \mathrm{~km} / \mathrm{h}$ & $6,0 \%$ \\
\hline
\end{tabular}

